\title{
Free Vibration of flexible soft-core sandwiches according to layerwise theories differently accounting for the transverse normal deformability
}

\author{
Ugo Icardi ${ }^{\mathbf{a}^{*}}$ (i) \\ Andrea Urracia \\ a Dipartimento di Ingegneria Meccanica e Aerospaziale - Politecnico di Torino - Corso Duca degli Abruzzi 24, 10129 Torino, Italy. E-mail: \\ ugo.icardi@polito.it, andrea.urraci@polito.it
}

* Corresponding author

https://doi.org/10.1590/1679-78255624

\begin{abstract}
This study aims to generalize a previously developed accurate and inexpensive 3-D zig-zag theory up to an arbitrary representation form and to determine which simplifications are yet accurate in determining transverse shear and normal stress/deformation effects on vibrations of soft-core sandwiches with not moving middle/neutral plane (pumping). Natural frequencies are calculated using displacements assumed differently across the thickness, having fixed d.o.f., not yet explored forms of representation and zig-zag functions differently accounting for the transverse normal deformability and that partially or fully fulfill physical constraints. Applications are presented for sandwich plates and beams with length-to-thickness ratios and material properties of faces and core varying within an industrial range, for which layerwise effects are very important and so suited to the evaluation of theories. Analytical solutions are found using the same trial functions and expansion order for all theories, so to evaluate their accuracy under the same conditions. The choice of the representation form and of zig-zag functions is shown immaterial if displacement field coefficients are recomputed across the thickness by enforcing the fulfillment of all physical constraints (using symbolic calculus). Furthermore, it is shown that assigning a specific role to each coefficient is immaterial, as well as exchanging the order of representation of in-plane and transverse displacement components and even that zig-zag functions could be omitted. This no longer occurs for lower-order theories with only a partial fulfillment of constraints. Pumping motions are highlighted as the first modes, which require the theories much accurately accounting for transverse normal deformability.
\end{abstract}

\section{Keywords}

Composite and sandwich plates and beams, interlaminar transverse shear and normal stress continuity, Navier's and 3D finite element analysis (FEA) solutions, free vibration behavior, different zig-zag functions, various form of representation of variables

\section{INTRODUCTION}

As it is well known, sandwiches find continuously increasing applications in aerospace, naval and terrestrial applications owing to superior specific strength and stiffness than traditional materials. 
Table 1 Acronyms.; in bold the new teories; ${ }^{(n)}$ degree of displacements.

\begin{tabular}{|c|c|c|c|}
\hline Acronym & Description (section) & Acronym & Description (section) \\
\hline FEA-3D & Mixed solid elements (Icardi and Atzori (2004)). & MHR4 & $\begin{array}{l}\text { Mixed HR theory, displacements with Murakami's zig- } \\
\text { zag function (Icardi and Urraci }(2018 b))^{(3.3)} \text {. }\end{array}$ \\
\hline FSDT & $\begin{array}{l}\text { Equivalent first-order shear deformation theory, } \\
\text { shear correction factor of } 5 / 6 \text { is assumed. }\end{array}$ & MHR \pm & $\begin{array}{l}\text { MHR theory, where the right sign of Murakami's zig-zag } \\
\text { function is determined at each interface on a physical } \\
\text { basis, (Icardi and Urraci }(2018 a))^{(3.3)} \text {. }\end{array}$ \\
\hline HRZZ & $\begin{array}{c}\text { Mixed HR theory, } u_{\alpha, \beta}^{(3)}, u_{\varsigma}^{(0)},(\text { Icardi and Urraci } \\
(2018 \mathrm{~b}))^{(3.3)} \text {. }\end{array}$ & MHR4 \pm & $\begin{array}{l}\text { MHR4 theory, where the right sign of Murakami's zig- } \\
\text { zag function is determined at each interface on a } \\
\text { physical basis (Icardi and Urraci (2018a) })^{(3.3)} \text {. }\end{array}$ \\
\hline HRZZ4 & $\begin{array}{c}\text { Mixed HR theory, } u_{\alpha, \beta}^{(3)}, u_{\varsigma}^{(4)}, \text { (Icardi and Urraci } \\
(2018 \mathrm{~b}))^{(3.3)} .\end{array}$ & MHWZZA & $\begin{array}{l}\text { Mixed HW theory, displacements with Murakami's zig- } \\
\text { zag functions, stress and strains with Di Sciuva's one } \\
\text { (Icardi and Urraci (2018b) })^{(3.3)}\end{array}$ \\
\hline HSDT & $\begin{array}{l}\text { Equivalent third-order shear deformation } \\
\text { theory. }\end{array}$ & MHWZZA4 & $\begin{array}{l}\text { Mixed HW theory, in-plane displacements with } \\
\text { Murakami's zig-zag functions, transverse displacement, } \\
\text { stress and strains with Di Sciuva's one (Icardi and Urraci } \\
(2018 \mathrm{~b}))^{(3.3)} \text {. }\end{array}$ \\
\hline HSDT_32 & $\begin{array}{l}\text { Enriched versions of HSDT, whose coefficients } \\
\text { are redefined across the thickness, } u_{\alpha, \beta}^{(3)}, u_{\varsigma}^{(2)} \\
\text { (4.4). }\end{array}$ & PP23 & $u_{\alpha, \beta}^{(2)}, u_{\varsigma}^{(3)}$ zig-zag theory (Icardi (2001)) $)^{(3.3)}$. \\
\hline HSDT_33 & $\begin{array}{l}\text { Enriched versions of HSDT, whose coefficients } \\
\text { are redefined across the thickness, } u_{\alpha, \beta}^{(3)}, u_{\varsigma}^{(3)} \\
\text { (4.4). }\end{array}$ & $\mathrm{ZZ}$ & $u_{\alpha, \beta}^{(3)}, u_{\varsigma}^{(4)}$ zig-zag theory (Icardi (2001)) $)^{(3.3)}$. \\
\hline HSDT_34 & $\begin{array}{l}\text { Enriched versions of HSDT, whose coefficients } \\
\text { are redefined across the thickness, } u_{\alpha, \beta}^{(3)}, u_{\varsigma}^{(4)} \\
\text { (4.4). }\end{array}$ & ZZA & $\begin{array}{l}u_{\alpha, \beta}^{(3)}, u_{\varsigma}^{(4)} \text { zig-zag adaptive theory (Icardi and Sola } \\
\qquad(2014))^{(2.2)}\end{array}$ \\
\hline HWZZ & $\begin{array}{l}\text { HW mixed version of ZZA (Icardi and Urraci } \\
\qquad(2018 b))^{(2.4)}\end{array}$ & ZZA* & $u_{\alpha, \beta}^{(3)}, u_{\varsigma}^{(4)}$ modified ZZA (Icardi and Urraci (2018a))(2.3). \\
\hline HWZZ_RDF & Modified HWZZ theory ${ }^{(4.2)}$. & ZZA*_43 & $u_{\alpha, \beta}^{(4)}, u_{\varsigma}^{(3)}$ modified version of ZZA* (4.3). \\
\hline HWZZM & $\begin{array}{l}\text { HWZZ with different zig-zag functions (Icardi and } \\
\text { Urraci (2018a)) })^{(3)} \text {. }\end{array}$ & $\begin{array}{l}\text {ZZA_X1 } \\
Z Z A \_X 2 \\
Z Z A \_X 3 \\
Z Z A \_X 4\end{array}$ & $\begin{array}{l}u_{\alpha, \beta}^{(3)}, u_{\varsigma}^{(4)} \text { modified ZZA theories, with different } \\
\quad \text { representation across the thickness }{ }^{(4.1)} \text {. }\end{array}$ \\
\hline HWZZM* & $\begin{array}{l}\text { HWZZ with different zig-zag functions (Icardi and } \\
\text { Urraci (2018a)) })^{(3.2)} \text {. }\end{array}$ & ZZA-XX & $\begin{array}{l}\text { Zig-zag general theory with exponential } \\
\text { representation }{ }^{(4.1)} \text {. }\end{array}$ \\
\hline HWZZM (ه) & $\begin{array}{l}\text { HWZZM theories, type A, B, B2, C, C2, 0, (Icardi } \\
\text { and Urraci (2018a) })^{(3.1)} \text {. }\end{array}$ & $Z Z A-X X^{\prime}$ & Zig-zag general theory with power representation ${ }^{(4.1)}$. \\
\hline MHR & $\begin{array}{l}\text { Mixed HR theory } u_{\alpha, \beta}^{(3)} \text { with Murakami's zig-zag } \\
\text { function, } u_{\varsigma}^{(4)} \text {, lacking (Icardi and Urraci } \\
(2018 \mathrm{~b}))^{(3.3)} .\end{array}$ & ZZA_RDF & Modified ZZA theory ${ }^{(4.1)}$. \\
\hline
\end{tabular}

However, their displacement field is no longer C1-continuous as in homogeneous non-layered materials, but instead it is $\mathrm{C}^{\circ}$-continuous (zig-zag effect) because only appropriate slope discontinuities at interfaces allow the fulfilment of local equilibrium equations, namely the continuity of transverse shear and normal stresses and of the transverse normal stress gradient at layer interfaces.

A multitude of theories have been proposed so far for multilayered structures, to which sandwiches are ascribed whenever it is not necessary to describe cell scale effect of honeycomb core, which can be summarily categorized into equivalent single-layer (ESL), discrete-layer (DL) and zig-zag (ZZ) theories (acronyms used throughout the paper are defined in Table 1).

They can further be subdivided into displacement-based and mixed theories, as strains and stresses could be chosen separately from one another using Hellinger-Reissner (HR) and Hu-Washizu variational theorems (HW). The papers by Carrera (1999, 2001, 2003, 2004), Carrera and Ciuffreda (2005), Demasi (2004), Vasilive and Lur'e (1992), 
Reddy and Robbins (1994), Lur'e, and Shumova (1996), Noor et al. (1996), Altenbach (1998), Khandan et al. (2012) and Kapuria and Nath (2013) and the book by Reddy (2003) are cited among many others, wherein a broad discussion of this matter can be found. However a brief description must be given to justify accuracy and computational costs that differ greatly.

ESL, completely disregard layerwise effects and are cheap but also unable to accurately predict through-thickness displacement and stress fields and even overall response quantities for certain loading, material properties and stackup and for sandwiches, as shown e.g. by Zhen and Wanji (2006), Kapuria et al. (2004), Burlayenko et al. (2015) and by Jun et al. (2016), by analyses carried out by Carrera's unified formulation (CUF) (see Carrera (2001)) and by advanced ZZ (see Icardi and Sola (2014), Icardi and Urraci (2018a, 2018b)). Also DL, which assume quantities at layer level as the functional d.o.f., are of limited validity since their use must be restricted to limited regions of structures of industrial interest, otherwise the computational capacity could be overwhelmed due to too many unknowns growing with the number of physical and computational layers considered. It must however be recognized that DL have the merit of accurately predicting displacement and stress fields irrespective for lay-up, layer properties, loading or boundary conditions considered. ZZ theories, having intermediate features between ESL and DL, strikes the right balance between accuracy and cost saving, allowing designers' demand of theories in a simple already accurate form to be met. Often higher-order sandwich theories are considered wherein in-plane and transverse displacements vary nonlinearly across the thickness and takes different forms between the faces and the core.

One of the first examples of higher-order sandwich theory for dynamic studies is the paper by Cho et al. (1991) wherein similarly to DL a separate representation is used for each constituent layer. The results of this paper show that natural frequencies are overestimated by ESL for laminates with arbitrary layouts and soft-core sandwiches. Besides it is shown that sandwiches can vibrate even at lower frequencies in modes where the middle plane does not move (pumping), which so cannot be captured by any lower order theory not properly accounting for transverse normal deformability effects.

Higher-order theories are of interest for industrial applications, such as structural health monitoring techniques for revealing and locating damage that are based on guided-wave propagation across the thickness (Barouni and Saravanos (2016)). The structural models used for studying through-thickness vibrations often consider the face sheets as ordinary plates with flexural and in-plane rigidities, irrespective they are made by different plies, although studies carried out by accounting for the laminated construction, including the present one, show that low frequency pumping occurs more easily when the faces consist of a superposition of rigid and less rigid materials. The core is often assumed to be shear resistant in the transverse direction, free of in-plane normal and shear stresses and deformable in the thickness direction.

One of the first studies in this field is published by Frostig and Thomsen (2004), where free vibration throughthickness modes of simply-supported sandwiches with equal faces are investigated using two different structural models. The first assumes the core transverse shear stress and the displacements of the faces as unknowns, while the other assumes a polynomial displacement field across the core based on the former one, whose unknowns are in additions the coefficients of polynomials. The latter model is suited for capturing nonlinear distributions of vertical and in-plane deformations, which are important when the core is soft and flexible. Another example where the same vibration problem is studied is the paper by Rao and Desai (2004), wherein analytical solutions are obtained using equivalent single layer and mixed layerwise theories. The latter ones account for warping of transverse cross-section. In a subsequent article, Malekzadeh et al (2005) investigate the dynamic behavior of sandwiches with a soft viscoelastic flexible core using non-linear displacements across the thickness, so to account for the distortions of the crosssectional plane and for the transverse normal deformation, while the face sheets are described by FSDT.

Schwarts-Givli et al. (2007) study the free vibration of delaminated unidirectional sandwich panels using a structural model accounting for the flexibility of the core in the thickness direction and assuming nonlinear displacement, acceleration, and velocity fields across it. More recently, Frostig and Thomsen (2009a, 2009b) carried out with a flexible and compliant core in the vertical direction and with temperature-dependent mechanical properties. In another recent paper, Lopatin and Morozov (2010) investigate pumping vibrations of sandwiches with equal faces considering compressive and shear stiffnesses and a nonlinear distribution of the transverse normal displacement across the core thickness. Rahmani et al. (2010) study the free vibration behavior of a composite sandwich cylindrical shell with a flexible core using the classical shell theory for the face sheets and an elasticity approach for the core, so to account for high-order effects caused by the nonlinearity of in-plane and transverse displacements.

Numerous studies of soft-core sandwiches with laminated faces have been recently carried out expanding the goals of analysis beyond global quantities, that is to through-thickness modal distributions of displacements and stresses. Those wherein sandwiches are described as three-layers structures have been abandoned in favour of 
layerwise theories which allow to represent each face ply as a single physical layer and the core is splitted into one or more mathematical layers. Last in order of time, the paper by Yang et al. (2017) constitutes an example where a closedform free vibration analysis of simply supported composite laminated beams has been carried out employing Carrera Unified Formulation (CUF) (Carrera (2001)) which can solve cases including general loading and boundary conditions where other theories cease to be accurate (that is why it is today extensively applied). CUF allows displacements to take arbitrary forms that can be chosen by the user as an input of the analysis and also gets existing ESL and Murakami's zig-zag theories (MZZ, discussed below) as particularizations. But also refined ZZ theories (Icardi and Sola (2014), Icardi and Urraci (2018a, 2018b)) allow to overcome certain limitations of the theories of sandwiches, such as the assumption of faces of equal thickness, with the same elastic properties and described as a single equivalent layer. Moreover it must be stressed that refined ZZ allow obtaining an accuracy comparable to that of CUF with fewer variables. Papers by Icardi and Urraci $(2018 a, 2018 b)$ represent successful attempts to extend the physically-based ZZ formulation to assume a degree of generalization comparable to that of CUF. This research continues in this paper through the evolution of ZZ towards a physically-based general theory that considers arbitrary displacement fields and that does not require to embed zig-zag functions explicitly, the displacement coefficients being redefined for each physical or computational lamina, which requires a computational burden still comparable to that of ESL. As a necessary premise to better frame the new contributions brought in this paper, a brief discussion of the characteristics of available ZZ follows.

ZZ can be subdivided into physically-based (DZZ) and kinematic-based (MZZ) theories because layerwise contributions are expressed differently. In DZZ they are embodied as the product of linear (Di Sciuva (1984)) or nonlinear (Icardi (2001)) zig-zag functions and unknown zig-zag amplitudes, while they are a priori assumed as functions featuring a periodic change of the slope of displacements at interfaces in MZZ, then stress fields are assumed apart from displacements within the framework of Hellinger-Reissner mixed variational theorem. The reason why MZZ theories sometimes provide an inappropriate representation of the displacement field (Icardi and Urraci (2018b)) is that the slope of displacements does not necessarily invert at interfaces as instead it is forcibly assumed. In addition there is also the possibility that while stresses are accurately reproduced, displacements are not because of the simplified kinematic assumptions used very often.

Improved DZZ based on a global-local superposition of displacement fields, have been proposed by Li and Liu (1997) and refined over the years by Zhen and Wanji (see e.g. Zhen and Wanji (2007)). ZZA theory by Icardi and Sola (2014) and those by Icardi and Urraci (2018a, 2018b) as its generalizations have five fixed d.o.f. and through-thickness variable kinematic representation that satisfies physical and elasticity theory constraints. They proved capable of the same accuracy of CUF and DL with a lower number of variables, as proven in Icardi and Urraci (2018b). The representation can be arbitrarily chosen by the user to obtain a redefinition of the coefficients of the displacement field similar to what would get by DL. Although preliminary, the study by Icardi and Urraci (2018b) shows that physically-based zig-zag theories with feature similar to those of CUF and HT (discussed below) can be developed starting from different assumptions and by considering forms of representation different for each displacements, that also vary across the thickness. Not yet explored zig-zag functions have also been considered, as will as theories wherein they are even omitted (and therefore with an additional cost benefit), provided that a number of coefficients equal to the number of stress compatibility conditions (or, in general, of constraints enforced) is recomputed at interfaces.

Theories based on a hierarchical set of locally defined polynomials (HT) have been developed (see, Catapano et al. (2011) and de Miguel et al. (2018)), which neither incorporate zig-zag contributions, nor post-processing steps, but which require a large number of degrees of freedom and a large expansion order. As an expamle of the studies where a variable-kinematics is used, see e.g. Vescovini and Dozio (2016). Although dynamic studies are still overwhelmingly carried out using ESL, excluding those previously mentioned concerning sandwiches, those carried out using DL and ZZ and CUF have highlight the superior performance of refined theories (see, e.g., Vescovini and Dozio (2016), Boscolo and Banerjee (2014), Khdeir and Aldraihem (2016), Sayyad and Ghugal (2015), Kazancı (2016), Lin and Zhang (2018)). In particular, those accounting for the transverse normal deformability have shown such deformation important even to predict first free-vibration modes of sandwiches with flexible soft-core, as highlighted in the previously cited articles (Cho et al. (1991), Barouni and Saravanos (2016), Frostig and Thomsen (2004, 2009a, 2009b), Rao and Desai (2004), Malekzadeh et al (2005), Schwarts-Givli et al. (2007), Lopatin and Morozov (2010), Rahmani et al. (2010), Yang et al. (2017)).

Further research is required to get even whether and when CUF and refined DZZ of Icardi and Urraci (2018b) can provide a more accurate information than approaches based on a three-layer description. In particular, it is necessary to verify with which geometrical and material properties and boundary conditions this can occur (e.g. when face properties are asymmetric due to different geometric and material properties). It must also be better clarified what is 
the minimum order and type of representation that allows to capture these effects. In this context it is necessary to test more extensively than in Icardi and Urraci (2018b) if theories derived from ZZA without the explicit incorporation of the zig-zag functions, or with arbitrary choices of these functions, as well as with arbitrary displacements are actually always as accurate as the parent theory.

It must be extensively tested even if theories derived from ZZA with a partial a priori assumption of zig-zag functions, which of consequence doesn't satisfy certain physical constraints, may still be sufficiently accurate. To contribute to clarify this matter, in this paper various theories derived by the authors as variants of ZZA Icardi and Sola (2014) that are obtained assuming differently the representation of displacement components one from the other and in different sections across the thickness, as well as differently assuming zig-zag functions or even omitting them are developed and compared each other, to reference results retaken from literature and to 3-D FEA (Icardi and Atzori (2004)). The purpose of numerical illustrations is to confirm the findings of previous articles (Icardi and Sola (2014), Icardi and Urraci (2018a, 2018b)), namely that the choice of zig-zag functions is immaterial, that these functions can even be omitted when a sufficient number of coefficients is incorporated that are re-determined at interfaces, that displacements can be arbitrarily chosen for the dynamic cases considered, provided that the full set of physical constraints (Khandan et al. (2012), Reddy (2003), Zhen and Wanji (2006), Kapuria et al. (2004)) is satisfied, as discussed below.

The intended aim of numerical applications is also to show how a partial fulfillment of these constraints reflects on accuracy of results, as well as that the redefinition of coefficients which is consequent to the imposition of constraints allows theories to significantly increase their accuracy. The aim is also to show that there is no variation in the accuracy of results exchanging the order of representation of in-plane and transverse displacement components. Furthermore it is proven that for lower-order theories with only a partial fulfillment of constraints the choice of zig-zag and representation functions can no longer be arbitrary and which is the lowest degree and order of expansion that is sufficient to capture the pumping modes. Furthermore it is proven that assigning a specific role a priori to the displacement field coefficients is unnecessary as their role can be freely exchanged without changing the results. To prove all these claims, the theories of this paper are applied to the study of free vibrations of flexible soft-core sandwich plates, for which the transverse deformation is as important as transverse shear one.

\section{THEORETICAL FRAMEWORK}

The feature of theories retaken from recent papers by the authors and others new proposed in this paper that are used in the numerical applications are presented and discussed below, so to provide the necessary theoretical support as well as to better understand meaning, distinctive features and limitations of the assumptions made and justify how they are reflected on accuracy of results. Those developed in this paper intend to demonstrate that the choice of zigzag functions is immaterial once physical constraints specified below are enforced, as well as also to show that their explicit incorporation is inessential. Indeed, interlayer stress compatibility conditions can be satisfied by the normal coefficients that describe the displacement field, once they are redefined across the thickness by imposing the appropriate physical constraints, as outlined in the following.

First the notations used and the basic assumptions (common for all the theories) are defined. Constituent layers are assumed to have linear elastic properties, a uniform arbitrary thickness $h^{k}$ and to be perfectly bonded to each other (bonding resin interlayer disregarded). Global-scale response being examined, cell-scale effects are also disregarded, so sandwiches are described in homogenized form as multi-layered structures with a thick soft intermediate layer as the core.

As reference frame, a rectangular, right-handed Cartesian coordinate reference system $(x, y, z)$ is assumed, $(x, y)$ being on the middle reference plane $\Omega$ of the multilayered plate (origin in the lower left edge) and $z$ as the thickness coordinate $\left(z \in\left[-\frac{h}{2}, \frac{h}{2}\right], h\right.$ being the overall thickness and $L x$ and $L y$ being the plate side-lengths). ${ }^{(k)} z^{+}$and ${ }^{(k)} z^{-}$ represents the upper and lower position of interfaces, respectively, subscripts $\mathrm{k}$ and superscripts ${ }^{\mathrm{k}}$ are used to indicate the belonging of quantities to the layer $k$, while " and I mark upper and lower faces of the laminate. Symbols $x, y, z$ are replaced by greek letters, i.e. $\alpha=1,2 \equiv x, y ; \varsigma=3 \equiv z$, to make formulas compact.

Elastic in-plane and transverse displacement components are indicated as $u_{\alpha}$ and $u_{\varsigma}$, respectively, a comma is used to indicate spatial derivatives (e.g., $\left.(.)_{, x}=\partial / \partial x,(.)_{, z}=\partial / \partial z\right)$ and strains are assumed to be infinitesimal. Strains and stresses are symbolized by $\varepsilon_{i j}$ and $\sigma_{i j}$, respectively. All theories assume the middle-plane displacements 
$u^{0}, v^{0}, w^{0}$ and the rotations of the normal $\theta_{x}=\Gamma_{x}^{0}(x, y)-w^{0}(x, y)_{, x}, \theta_{y}=\Gamma_{y}^{0}(x, y)-w^{0}(x, y)_{, y}$, as the only functional degrees of freedom. Governing dynamic equations will not be reported as they can be obtained in a straightforward way for each theory using standard variational techniques, so just displacement, strain and stress fields which are ones that affect accuracy will be discussed.

\subsection{Analytical solutions}

Dynamic governing equation are solved as customary expressing functional degrees of freedom (d.o.f.) as the product of an harmonic function of time $e^{i \omega t}$ and a truncated series expansion of unknown amplitudes $A_{\Delta}^{i}$ and trial functions $\Re^{i}(x, y)$, which individually satisfy prescribed boundary conditions:

$$
\Delta=\sum_{i=1}^{m_{\Delta}} A_{\Delta}^{i} \Re^{i}(x, y)
$$

the symbol $\Delta$ being used to represent in turns the d.o.f. $u^{0}, v^{0}, w^{0}, \theta_{x}, \theta_{y}$, while mechanical boundary conditions are satisfied using Lagrange multipliers method. So, a number of unknown amplitudes $A_{\Delta}^{i}$ is determined in proportion to the number of boundary conditions enforced, while those remaining are determined deriving governing functionals with respect to still unknown amplitudes and equating to zero, the work of inertial forces

$$
\int_{V}\left[-b_{i} \partial u_{i}\right] d v=\int_{V}\left[-\rho \ddot{u}_{i} \partial u_{i}\right] d v
$$

being considered. An algebraic system is obtained, whose solution provides the numerical value of each amplitude. The trial functions adopted in each individual application and the expansion order used for each case are specified in Table 2. They are chosen in order to satisfy the following boundary conditions, representative of simply-supported edges (other boundary conditions have been considered in Icardi and Urraci (2018a) and Icardi and Urraci (2018b), so they are not replicated also in the present study):

$$
\begin{aligned}
& w^{0}(0, y)=0 ; w^{0}\left(L_{x}, y\right)=0 ; w^{0}(0, y)_{, x x}=0 ; w^{0}\left(L_{x}, y\right)_{, x x}=0 \\
& w^{0}(x, 0)=0 ; w^{0}\left(x, L_{y}\right)=0 ; w^{0}(x, 0)_{, y y}=0 ; w^{0}\left(x, L_{y}\right)_{, y y}=0
\end{aligned}
$$

at $x=0, x=L_{x}$ and $y=0, y=L_{y}$ for $z=0$, i.e. on the reference mid-plane.

\subsection{ZZA displacement-based theory}

The through-thickness displacement field of this theory (from which all other theories of this paper are particularized) is postulated as (Icardi and Sola (2014), Icardi and Urraci (2018a, 2018b)):

$$
\begin{aligned}
& u_{\alpha}(x, y, z)=\left[u^{0}(x, y)+z\left(\Gamma_{\alpha}^{0}(x, y)-w^{0}(x, y)_{, \alpha}\right)\right]_{0}+\left[F_{\alpha}^{u}(z)\right]_{i}+\left[\sum_{k=1}^{n_{i}} \Phi_{\alpha}^{k}(x, y)\left(z-z_{k}\right) H_{k}(z)+\sum_{k=1}^{n_{\Im}}{ }_{\alpha} C_{u}^{k}(x, y) H_{k}(z)\right]_{c} \\
& u_{\varsigma}(x, y, z)=\left[w^{0}(x, y)\right]_{0}+\left[F^{\zeta}(z)\right]_{i}+\left[\sum_{k=1}^{n_{i}} \Psi^{k}(x, y)\left(z-z_{k}\right) H_{k}(z)+\sum_{k=1}^{n_{i}} \Omega^{k}(x, y)\left(z-z_{k}\right)^{2} H_{k}(z)+\sum_{k=1}^{n_{\varsigma}} C_{\varsigma}^{k}(x, y) H_{k}(z)\right]_{c}
\end{aligned}
$$

Linear- $[\ldots]_{0}$, higher- $[\ldots]_{i}$ and layerwise $[\ldots]_{c}$ contributions are incorporated, whose meaning and purpose are as follows. $[\ldots]_{0}$ contains the only five functional degrees of freedom, while $[\ldots]_{i}$ can contain any combination of independent functions $\left[F_{\alpha}^{u}(z)\right]_{i}$ and $\left[F^{\zeta}(z)\right]_{i}$, which here are chosen as: 


$$
\begin{aligned}
& {\left[F_{\alpha}^{u}(z)\right]_{i}=\left[C_{\alpha}^{i}(x, y) z^{2}+D_{\alpha}^{i}(x, y) z^{3}+\left(O z^{4} \ldots\right)\right]_{i}=\left[{ }_{3}\left(\tilde{)}_{\alpha}\right]_{i}+\left[\left(O z^{4} \ldots\right)\right]_{i}\right.} \\
& {\left[F^{\zeta}(z)\right]_{i}=\left[b^{i}(x, y) z+c^{i}(x, y) z^{2}+d^{i}(x, y) z^{3}+e^{i}(x, y) z^{4}+\left(O z^{5} \ldots\right)\right]_{i}=\left[{ }_{4}(.)_{\zeta}\right]_{i}+\left[\left(O z^{5} \ldots\right)\right]_{i}}
\end{aligned}
$$

and of which Icardi (2001) is a particularization with a through-thickness fixed representation, namely higher-order contributions $\left[\left(O z^{4} \ldots\right)\right]_{i},+\left[\left(O z^{5} \ldots\right)\right]_{i}$ are characteristic of ZZA, while terms $\left.\left[{ }_{3}(.)_{\alpha}\right]_{i},{ }_{4}(.)_{\zeta}\right]_{i}$ are the same as in the previous theory.

\begin{tabular}{|c|c|c|c|c|c|c|}
\hline Case & Lay-up & Layer thickness & Material & $\mathrm{Lx} / \mathrm{h}$ & Ly/Lx & BCS \\
\hline$a^{\operatorname{Kim}(2007)}$ & {$[0 / 90 / 0]$} & {$\left[(\mathrm{h} / 3)_{3}\right]$} & {$\left[(\mathrm{m})_{3}\right]$} & $4,10,20$ & - & SS \\
\hline b Cho et al. (1991) & {$[0]$} & {$[\mathrm{h}]$} & {$[\mathrm{n}]$} & 10 & 1 & SSSS \\
\hline C Icardi and Urraci (2018a) $(*+\S)$ & {$[0 / 90 / 0 / 0 / 90]$} & {$[(\mathrm{h} / 24) 2 /(5 \mathrm{~h} / 12)]_{\mathrm{s}}$} & [01/o2/p/o1/o2] & 5 & 1 & SSSS \\
\hline$d(*+\S)$ & {$[0 / 90 / 0 / 0 / 90]$} & {$[(\mathrm{h} / 24) 2 /(5 \mathrm{~h} / 12)]_{\mathrm{s}}$} & [01/o2/q/o1/o2] & 5 & 1 & SSSS \\
\hline e $\left({ }^{*}+\S\right)$ & {$\left[\mathrm{O}_{5}\right]$} & {$[(\mathrm{h} / 24) 2 /(5 \mathrm{~h} / 12)]_{\mathrm{s}}$} & {$[\mathrm{r} 1 / \mathrm{r} 2 / \mathrm{s} / \mathrm{r} 1 / \mathrm{r} 2]$} & 4 & 1 & SSSS \\
\hline$f\left({ }^{*}+\S\right)$ & {$\left[0_{5}\right]$} & {$[(\mathrm{h} / 24) 2 /(5 \mathrm{~h} / 12)]_{\mathrm{s}}$} & {$[\mathrm{r} 1 / \mathrm{r} 2 / \mathrm{t} / \mathrm{r} 1 / \mathrm{r} 2]$} & 4 & 1 & SSSS \\
\hline$g\left({ }^{*}+\S\right)$ & {$\left[0_{6}\right]$} & $\begin{array}{c}{\left[(\mathrm{h} / 24)_{2} /(30 \mathrm{~h} / 48) /\right.} \\
\left.(10 \mathrm{~h} / 48) /(\mathrm{h} / 24)_{2}\right]\end{array}$ & {$[\mathrm{r} 1 / \mathrm{r} 2 / \mathrm{s} / \mathrm{u} / \mathrm{r} 1 / \mathrm{r} 2]$} & 4 & 1 & SSSS \\
\hline
\end{tabular}

Table 2a Reference pattern of all cases ; ${ }^{*} \S$ : Murakami's slope assumption not respected by $u_{\alpha}\left({ }^{*}\right), u_{\beta}(+)$ and $u_{\varsigma}(\S)$.

Table $\mathbf{2 b}$ Normalization, trial functions and expansion order for each case.

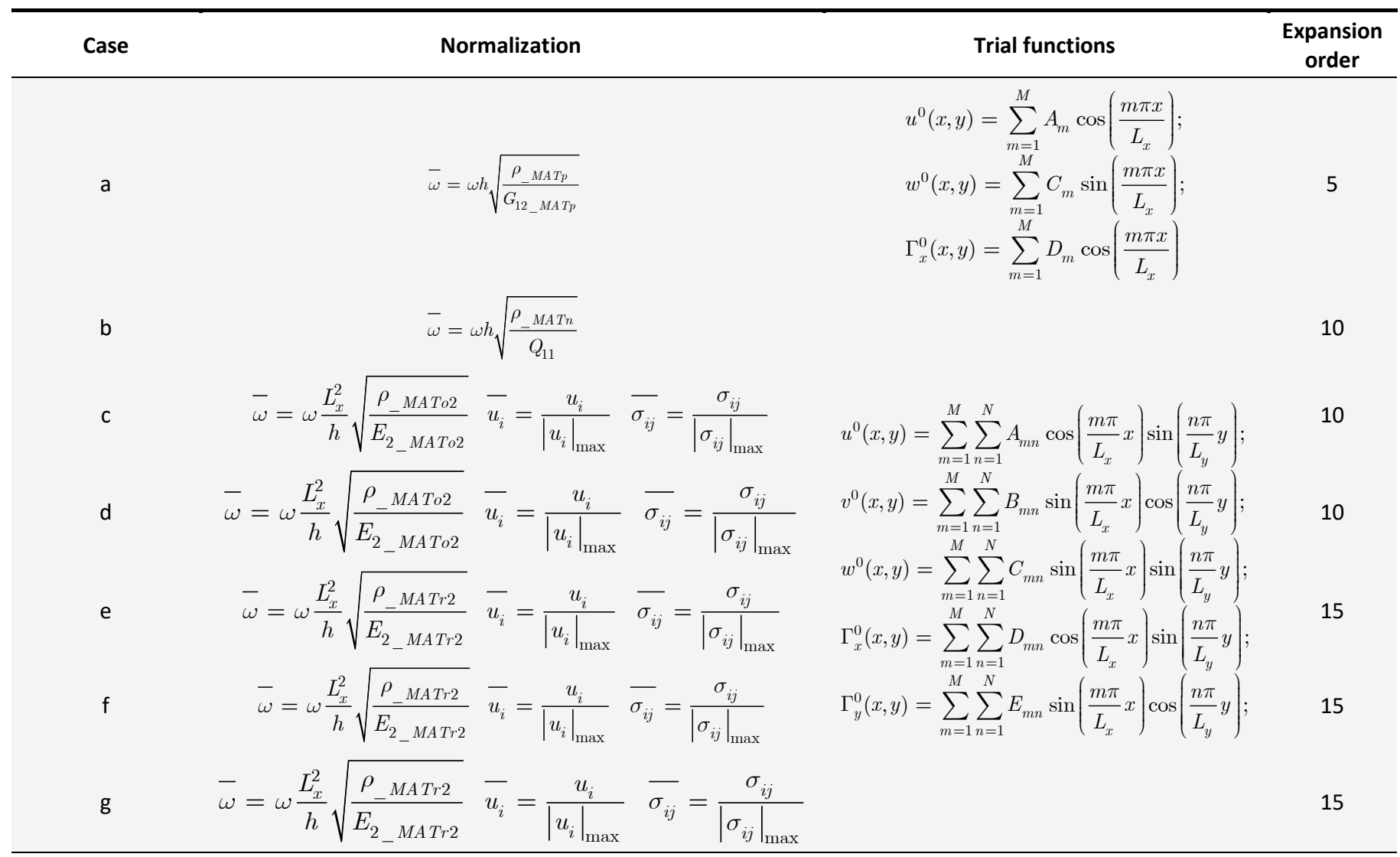

Note that $\left[F_{\alpha}^{u}(z)\right]_{i}$ and $\left[F^{\zeta}(z)\right]_{i}$ aren't just depending on $z$ because symbols ${ }_{\alpha}^{u},{ }^{\zeta}$ represent the functional dependence on the d.o.f. which are function of in-plane coordinates. Expressions of coefficients $C_{\alpha}^{i}, D_{\alpha}^{i}, b^{i}$ to $e^{i}$ are obtained using symbolic calculus by enforcing the fulfilment of stress boundary conditions 


$$
\sigma_{\alpha \varsigma}=\sigma_{\varsigma \varsigma, \varsigma}=0 ; \sigma_{\varsigma \varsigma}=p^{0}( \pm)
$$

having assumed only a transverse distributed loading to act at upper (+) and lower (-) faces (non-homogeneous conditions $\sigma_{\alpha \varsigma} ; \sigma_{\beta \varsigma} \neq 0$ could be enforced without difficulty). It should be noticed that for this paper, $p^{0}( \pm)=0$, being free vibration analyses. Contributions $[\ldots]_{i}$ by $(5)$ can be rearranged as:

$$
\begin{aligned}
& U_{\alpha}^{i}(x, y, z)=\left[A_{\alpha 2} z^{2}+A_{\alpha 3} z^{3}\right]+A_{\alpha 4} z^{4}+\ldots+A_{\alpha n} z^{n} \\
& U_{\zeta}^{i}(x, y, z)=\left[A_{\varsigma 1} z+A_{\varsigma 2} z^{2}+A_{\varsigma 3} z^{3}+A_{\varsigma 4} z^{4}\right]+A_{\varsigma 5} z^{5}+\ldots+A_{\varsigma n} z^{n}
\end{aligned}
$$

to highlight which those under square brackets are determined by enforcing the fulfilment of boundary conditions (6) and local equilibrium equations:

$\sigma_{\alpha \beta, \beta}+\sigma_{\alpha \varsigma, \varsigma}=b_{\alpha} ; \sigma_{\alpha \varsigma, \alpha}+\sigma_{\varsigma \varsigma, \varsigma}=b_{\varsigma}$

at selected points across the thickness, while higher-order contributions $A_{\alpha 4} z^{4}+\ldots+A_{\alpha n} z^{n}, A_{\varsigma 5} z^{5}+\ldots+A_{\varsigma n} z^{n}$, which enable a variable-kinematics representation across the thickness, are computed by imposing just the fulfilment of (8). Note that the in-plane position of equilibrium points can be chosen appropriately for each case and that coefficients that qualify displacements are re-computed specifically for each computational/physical layer, so that the representation can properly manage strong variations of layer properties without any increase of the d.o.f. In the numerical applications a third/fourth order representation that embraces the whole laminate is used being generally sufficient to obtain accurate results (Icardi and Sola (2014), Icardi and Urraci (2018a, 2018b)). [...] c represent layerwise contributions where the expressions of zig-zag amplitudes $\Phi_{\alpha}^{k}, \Psi^{k}$ and $\Omega^{k}$ are determined so that the continuity of out-of-plane stresses and of the transverse normal stress gradient $\sigma_{\varsigma \varsigma, \varsigma}$ at layer interfaces

$\sigma_{\alpha \varsigma}\left({ }^{(k)} z^{+}\right)=\sigma_{\alpha \varsigma}\left({ }^{(k)} z^{-}\right) ; \sigma_{\varsigma \varsigma}\left({ }^{(k)} z^{+}\right)=\sigma_{\varsigma \varsigma}\left({ }^{(k)} z^{-}\right) ; \sigma_{\varsigma \varsigma, \varsigma}\left({ }^{(k)} z^{+}\right)=\sigma_{\varsigma \varsigma, \varsigma}\left({ }^{(k)} z^{-}\right)$

is satisfied, as prescribed by the elasticity theory. The other layerwise contributions $C_{u}^{k}$ and $C_{\varsigma}^{k}$ restore the continuity of displacements

$u_{\alpha}\left({ }^{(k)} z^{+}\right)=u_{\alpha}\left({ }^{(k)} z^{-}\right) ; u_{\varsigma}\left({ }^{(k)} z^{+}\right)=u_{\varsigma}\left({ }^{(k)} z^{-}\right)$

at mathematical layer interfaces, i.e. when it is chosen to split physical layers. The symbols $n_{i}$ and $n_{\Im}$ in the summations of (4) are used to distinguish the number of physical layer interfaces from that of mathematical layer interfaces, respectively. The enforcement of equilibrium and stress compatibility conditions yields to a system of algebraic equations at each interface that is solved using a symbolic calculus tool. Notice that if just the material properties and/or the orientation of layers change, but not their number, symbolic expressions representing the solution will remain the same. The computation of zig-zag amplitudes takes only an small fraction of the overall calculation time, so costs remains compatible with that of ESL (see, Table 3). Note that SEUPT technique (Icardi and Sola (2014)) can be used to obtain a C formulation of the ZZA theory, as well as of all the other theories of this paper.

\subsection{ZZA* displacement-based theory}

A modified version of ZZA indicated as ZZA* (Icardi and Urraci (2018a)) is considered to show that zig-zag functions $\left(z-z_{k}\right) H_{k}$ and $\left(z-z_{k}\right)^{2} H_{k}$ ( $\mathrm{Hk}$ being the Heaviside unit step function: $\mathrm{Hk}=0$ for $\mathrm{z}<\mathrm{zk}$, while $\mathrm{Hk}=1$ for $\mathrm{z} \geq \mathrm{zk}$ ), can be replaced with any other arbitrary set of functions whose coefficients are redefined at layer interfaces through the enforcement of the whole set of physical constraints $(6,8-10)$, without the accuracy of the results being affected. This 
translates into the fact that contributions $[\ldots]_{c}$ can be completely omitted if other coefficients can be redefined across the thickness, even if they are not multiplied by zig-zag functions. As this latter choice speeds-up the computations of coefficients, it turns into a computational advantage that grows with the number of computational layers. To construct ZZA* $^{*}$, the displacement field (4) is rewritten as:

$$
\begin{aligned}
& \left.u_{\alpha}(x, y, z)=\left[u^{0}(x, y)+z\left(\Gamma_{\alpha}^{0}(x, y)-w^{0}(x, y)\right)_{\alpha}\right)\right]_{0}+\left\{\sum_{k=1}^{n_{\text {S }}}{ }_{k} \tilde{B}_{\alpha}^{i}(x, y) z++\left[C_{\alpha}^{i}(x, y) z^{2}\right]+\left[D_{\alpha}^{i}(x, y) z^{3}+D_{\alpha}^{i}(x, y) z_{j}^{3}\right]+\sum_{k=1}^{n_{i}}{ }_{k} \tilde{C}_{\alpha}^{i}(x, y)\right\}_{i+c} \\
& u_{\varsigma}(x, y, z)=\left[w^{0}(x, y)\right]_{0}+\left\{\left[b^{i}(x, y) z+\sum_{k=1}^{n_{3}}{ }_{k} \tilde{b}^{i}(x, y) z\right]+\left[c^{i}(x, y) z^{2}+\sum_{k=1}^{n_{i}}{ }_{k} \tilde{c}^{i}(x, y) z^{2}\right]+\left[d^{i}(x, y) z^{3}\right]+e^{i}(x, y) z^{4}+\sum_{k=1}^{n_{i}}{ }_{k} \tilde{d}^{i}(x, y)\right\}_{i+c}
\end{aligned}
$$

wherein $[\ldots]_{0}$ still contains the only five functional d.o.f., the same of ZZA. Terms ${ }_{k} \tilde{B}_{\alpha}^{i}, C_{\alpha}^{i},{ }_{k} \tilde{C}_{\alpha}^{i}$ serve the same purpose as $\Phi_{\alpha}^{k}, C_{\alpha}^{i}$ and ${ }_{\alpha} C_{u}^{k}$ inside ZZA, while ${ }_{k} \tilde{b}^{i}$ and ${ }_{k} \tilde{c}^{i}$ have the function of $\Omega^{k}$ and $\Psi^{k}$ and ${ }_{k} \tilde{d}^{i}$ that of $C_{\varsigma}^{k}$ once they are redefined across the thickness in order to satisfy physical constraints.

Table 3 Processing time [s] on a computer with quad-core CPU@2.60GHz, 64-bit OS and $8.00 \mathrm{~GB}$ RAM; errors: $\boldsymbol{\bullet}^{>} 3 \%$; $\boldsymbol{\bullet}^{\circ} 10 \%$.

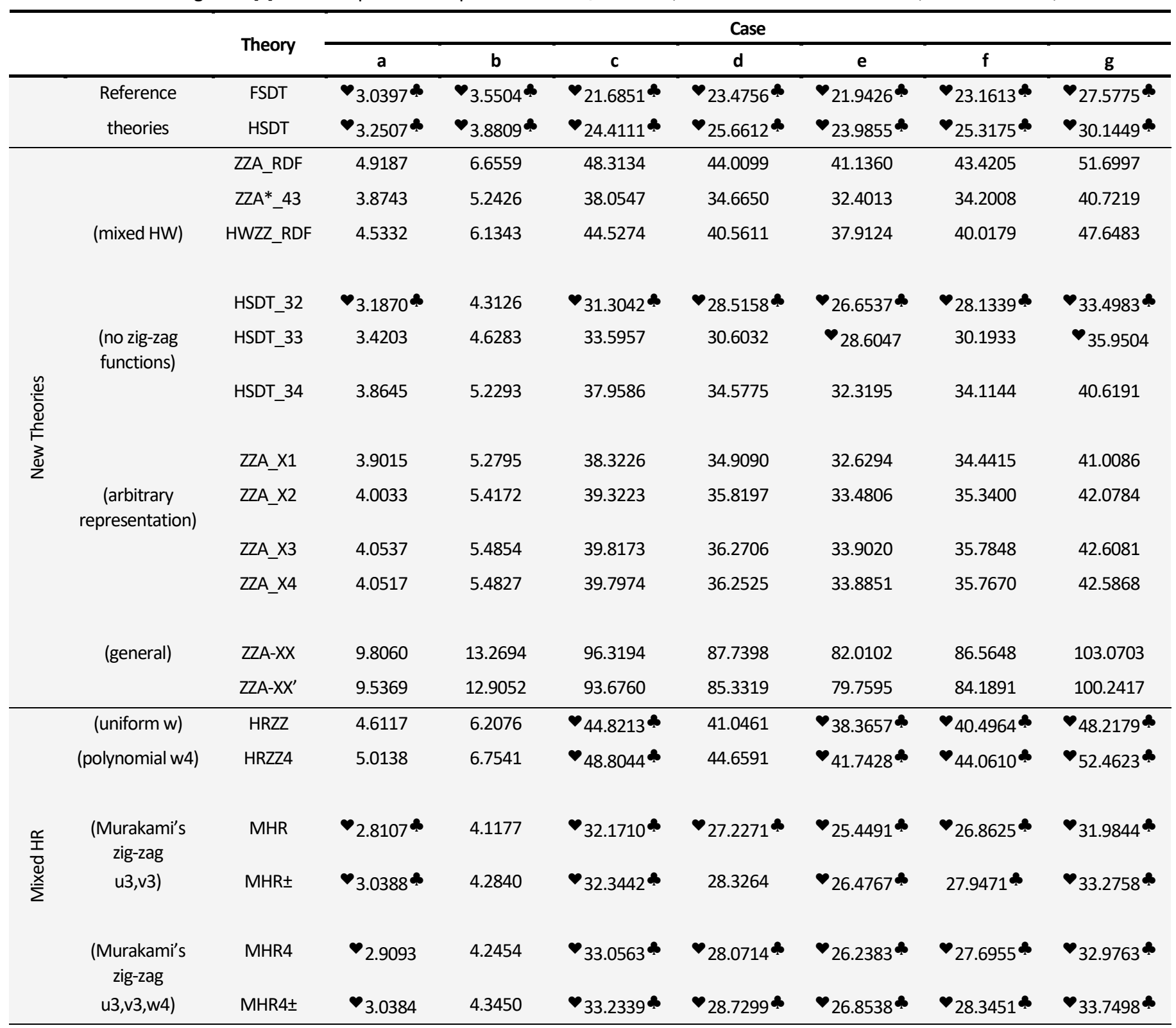




\begin{tabular}{|c|c|c|c|c|c|c|c|c|c|}
\hline & & \multirow{2}{*}{ Theory } & \multicolumn{7}{|c|}{ Case } \\
\hline & & & a & b & c & d & e & $f$ & g \\
\hline \multirow{15}{*}{ 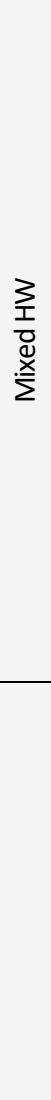 } & & HWZZ & 4.9640 & 6.4877 & 45.4263 & 42.8977 & 40.0964 & 42.3232 & 50.3931 \\
\hline & $\begin{array}{l}\text { (no zig-zag } \\
\text { functions) }\end{array}$ & HWZZM* & 3.9374 & 5.1532 & 36.1369 & 34.0740 & 31.8489 & 33.6177 & 40.0277 \\
\hline & & MHWZZA & $\boldsymbol{\bullet}_{3.7606^{\bullet}}$ & 5.1634 & $\boldsymbol{\bullet}_{38.0218}$ & $\boldsymbol{v}_{34.1415}$ & $\boldsymbol{\bullet}_{31.9120}$ & $\boldsymbol{v}_{33.6843}$ & $\boldsymbol{v}_{40.1070}$ \\
\hline & & MHWZZA4 & $\boldsymbol{\bullet}_{3.7602}$ & 5.1663 & $\boldsymbol{\bullet}_{38.0678}$ & $\boldsymbol{\bullet}_{34.1607}$ & $\boldsymbol{\bullet}_{31.9299}$ & $\boldsymbol{\bullet}_{33.7032}$ & $\boldsymbol{\bullet}_{40.1295}$ \\
\hline & & HWZZM & 4.1887 & 5.6511 & 40.8973 & 37.3664 & 34.9263 & 36.8660 & 43.8954 \\
\hline & $\begin{array}{l}\text { (Murakami's } \\
\text { zig-zag }\end{array}$ & HWZZMA & $\bullet_{4.1595^{\star}}$ & 5.5746 & $\bullet_{40.0733}$ & $\bullet_{36.8605^{\circ}}$ & $\bullet_{34.4534}$ & $\boldsymbol{v}_{36.3669}$ & $\bullet_{43.3010}$ \\
\hline & u3,v3,w4) & HWZZMB & $\boldsymbol{\bullet}_{4.1817}$ & 5.6134 & $\boldsymbol{P}_{40.4178}$ & 37.1167 & $\boldsymbol{P}_{34.6929^{\circ}}$ & 36.6197 & $\boldsymbol{v}_{43.6020^{\mathbf{p}}}$ \\
\hline & & HWZZMB2 & $\bullet_{4.1869}$ & 5.6175 & $\bullet_{40.4263}$ & $\bullet_{37.1439}$ & $\boldsymbol{\bullet}_{34.7183^{*}}$ & $\bullet_{36.6464}$ & $\bullet$ \\
\hline & & HWZZMC & 4.1659 & 5.6065 & 40.4737 & 37.0715 & 34.6507 & 36.5750 & 43.5489 \\
\hline & & HWZZMC2 & 4.1849 & 5.6148 & 40.4071 & 37.1262 & 34.7018 & 36.6290 & 43.6131 \\
\hline & & HWZZMO & 4.1554 & 5.5491 & $\boldsymbol{P}_{39.7426}$ & $\boldsymbol{\bullet}_{36.6915}$ & 34.2955 & $\bullet_{36.2002}$ & $\boldsymbol{v}_{43.1026}$ \\
\hline & $\begin{array}{l}\text { (adaptive } \\
\left.\mathrm{u}^{3}, \mathrm{v}^{3}, \mathrm{w}^{4}\right)\end{array}$ & ZZA & 5.3866 & 7.0182 & 48.9768 & 46.4055 & 43.3751 & 45.7840 & 54.5138 \\
\hline & $\left(u^{3}, v^{3}, w^{4}\right)$ & $\mathrm{ZZ}$ & $\boldsymbol{\bullet}_{3.7072}$ & 5.0165 & $\bullet_{36.4137}$ & 33.1702 & $\bullet_{31.0041}$ & $\boldsymbol{\bullet}_{32.7259}$ & $\boldsymbol{v}_{38.9659}$ \\
\hline & $\begin{array}{c}\text { (no zig-zag } \\
\text { functions) } \\
u^{3}, v^{3}, w^{4} \text { ) }\end{array}$ & $\mathrm{ZZA} *$ & 3.8722 & 5.2398 & 38.0345 & 34.6466 & 32.3841 & 34.1826 & 40.7003 \\
\hline & $\begin{array}{c}\text { (no zig-zag } \\
\text { functions) } \\
\mathrm{u}^{2}, \mathrm{v}^{2}, \mathrm{w}^{3} \text { ) }\end{array}$ & PP23 & $\boldsymbol{\nabla}_{2.8765^{\mathbf{s}}}$ & 3.8924 & $\boldsymbol{P}_{28.2542}$ & 25.7375 & $\boldsymbol{P}_{24.0568}$ & $\boldsymbol{\bullet}_{25.3928}$ & $\boldsymbol{v}_{30.2345^{2}}$ \\
\hline
\end{tabular}

Again $C_{\alpha}^{i}, D_{\alpha}^{i}, b^{i}, c^{i}, d^{i}$ and $e^{i}$ allow local equilibrium equations (8) and stress boundary conditions (6) and be met. At the bounding lower face $b^{i}$ and $c^{i}$ enable the fulfillment of stress boundary conditions regarding that transverse normal stress $\sigma_{\varsigma \varsigma}$ and gradient $\sigma_{\varsigma \varsigma, \varsigma}$ then they are canceled in subsequent layers where $C_{\alpha}^{i}, D_{\alpha}^{i}, d^{i}$ and $e^{i}$ allow to satisfy the three equilibria (8) at two points per layer (excluding the upper layer), while these coefficients enable stress-free boundary conditions on $\sigma_{\alpha \varsigma}$ and three equilibrium equations at a single point to be satisfied.

The remaining still free variables allow to meet three equilibrium equations at a single point across the upper layer and the boundary conditions at the upper bounding surface. It could be noticed that the first, the intermediate and the last layers can be subdivided each into two or more computational layers if more equilibrium points are required for improving accuracy. However, this decomposition into computational layers does not prove necessary for the benchmarks considered, just a third/fourth order overall piecewise representation of displacements being usually appropriate.

However for this to happen, the displacement field (11) must contain a sufficient number of contributions for ensuring the imposition of a sufficient number of equilibrium conditions at selected positions $z_{j}$ across the thickness. This doesn't necessarily mean that an increased order of expansion is required, because coefficients can be evaluated at different positions using the same power of the thickness coordinate.

Numerical illustrations will show that ZZA* can achieve the same accuracy of ZZA with a little lower computational effort, but the most important result is to demonstrate that the choice of zig-zag functions is immaterial, whenever coefficients are redefined layer-by-layer as outlined above (otherwise accuracy would depend on the choice of zig-zag functions). 


\subsection{HWZZ mixed theory}

HWZZ theory (Icardi and Urraci (2018b)) is a mixed version of ZZA developed within the framework of Hu-Washizu variational theorem which is obtained keeping only contributions to displacement, strain and stress fields deemed essential, for the purpose of reducing the computational effort but preserving accuracy.

Displacements derive from those of ZZA neglecting the contributions of $\Omega^{k}$ which provide much less important slope variations than $\Phi_{\alpha}^{k}, \Psi^{k}$. Higher-order and adaptive contributions, $A_{\alpha 4} z^{4}+\ldots+A_{\alpha n} z^{n}, A_{\varsigma 5} z^{5}+\ldots+A_{\varsigma n} z^{n}$, which as $\Omega^{k}$ are important for an accurate description of the stress field in ZZA, but here because stresses are assumed separately from displacements, are also neglected.

As a direct consequence, no decomposition into mathematical layers is allowed For consistency, consequently, contributions $C_{u}^{k}, C_{v}^{k}, C_{w}^{k}$ are omitted. As a result, the displacement field of HWZZ is:

$$
\begin{aligned}
& u_{\alpha}(x, y, z)=\left[u^{0}(x, y)+z\left(\Gamma_{x}^{0}(x, y)-w^{0}(x, y)_{, \alpha}\right)\right]_{0}+\left[C_{\alpha}^{i}(x, y) z^{2}+D_{\alpha}^{i}(x, y) z^{3}\right]_{i}+\left[\sum_{k=1}^{n_{i}} \Phi_{\alpha}^{k}(x, y)\left(z-z_{k}\right) H_{k}(z)\right]_{c} \\
& u_{\varsigma}(x, y, z)=\left[w^{0}(x, y)\right]_{0}+\left[b^{i}(x, y) z+c^{i}(x, y) z^{2}+d^{i}(x, y) z^{3}+e^{i}(x, y) z^{4}\right]_{i}+\left[\sum_{k=1}^{n_{i}} \Psi^{k}(x, y)\left(z-z_{k}\right) H_{k}(z)\right]_{c}
\end{aligned}
$$

The decomposition into mathematical layers is restored in out-of-plane strains $\varepsilon_{z z}, \gamma_{x z}, \gamma_{y z}$, as their expressions are derived from

$$
\begin{aligned}
& { }^{\Im} u_{\alpha}(x, y, z)=\left[u^{0}(x, y)+z\left(\Gamma_{x}^{0}(x, y)-w^{0}(x, y)_{, \alpha}\right)\right]_{0}+\left[C_{\alpha}^{i}(x, y) z^{2}+D_{\alpha}^{i}(x, y) z^{3}\right]_{i}+\left[\sum_{k=1}^{n_{i}} \Phi_{\alpha}^{k}(x, y)\left(z-z_{k}\right) H_{k}(z)+\sum_{k=1}^{\Im}{ }_{\alpha} C_{u}^{k}(x, y) H_{k}\right]_{c} \\
& { }^{\Im} w_{\varsigma}(x, y, z)=\left[w^{0}(x, y)\right]_{0}+\left[b^{i}(x, y) z+c^{i}(x, y) z^{2}+d^{i}(x, y) z^{3}+e^{i}(x, y) z^{4}\right]_{i}+\left[\sum_{k=1}^{n_{i}} \Psi^{k}(x, y)\left(z-z_{k}\right) H_{k}(z)+\sum_{k=1}^{\Im} C_{w}^{k}(x, y) H_{k}\right]_{c}
\end{aligned}
$$

The symbol ${ }^{\Im}($.$) states that displacements refer to the computational layer \Im$. Instead, no decomposition into mathematical layers is allowed for the in-plane strains, they being derived from (12).

The expressions of membrane stresses $\sigma_{x x}, \sigma_{y y}, \sigma_{x y}$ are obtained in a straightforward way from stress-strain relations, while for improving accuracy and recovering mistakes consequent the restrictive assumptions made, such as the small stress jumps resulting from omission of contributions by $\Omega^{k}, \sigma_{x z}, \sigma_{y z}, \sigma_{z z}$ are obtained from membrane stresses by integrating local equilibrium equations (8).

\section{HWZZM MIXED THEORY}

It is constructed following a similar pattern to HWZZ in order to show that the choice of zig-zag functions is immaterial, as the same results can be achieved irrespective of the choice made whenever the full set of physical constraints discussed so far in this paper are enforced. HWZZM is developed assuming the following displacement field (Icardi and Urraci (2018a)):

$$
\begin{aligned}
& { }^{\Im} u_{\alpha}(x, y, z)=\left[u^{0}(x, y)+z\left(\Gamma_{\alpha}^{0}(x, y)-w^{0}(x, y)_{, \alpha}\right)\right]_{0}+\left[F_{\alpha}^{u}(z)\right]_{i}+\left[A_{k}^{u_{\alpha}}(z)\left[\frac{2 z}{z_{k+1}-z_{k}}-\frac{z_{k+1}+z_{k}}{z_{k+1}-z_{k}}\right]+C_{\alpha}^{k}(x, y)\right]_{c} \\
& { }^{\Im} u_{\varsigma}(x, y, z)=\left[w^{0}(x, y)\right]_{0}+\left[F^{\zeta}(z)\right]_{i}+\left[A_{k}^{u_{\varsigma}}(z)\left[\frac{2 z}{z_{k+1}-z_{k}}-\frac{z_{k+1}+z_{k}}{z_{k+1}-z_{k}}\right]+B_{k}^{u_{\varsigma}}(z)\left[\frac{(2 z)^{2}}{z_{k+1}-z_{k}}\right]+C_{\varsigma}^{k}(x, y)\right]_{c}
\end{aligned}
$$

Layerwise functions within the third square bracket constitute variations of Murakami's zig-zag contributions $M^{k}(z)=(-1)^{k} \zeta^{k}$ in canonical form, where $\zeta^{k}=a^{k} z-b^{k}, a^{k}=\frac{2}{z_{k+1}-z_{k}}, b^{k}=\frac{z_{k+1}+z_{k}}{z_{k+1}-z_{k}}$, which, unlike what done 
in the literature, are multiplied by amplitudes $A_{k}^{u_{\varsigma}}$ and $B_{k}^{u_{\varsigma}}$ whose expressions are defined by enforcing the fulfillment of interfacial stress compatibility conditions.

Note that this is operation is the one making the choice of zig-zag functions immaterial, since the product of zigzag amplitudes and functions becomes an invariant, as shown by the numerical results, once the full set of physical stress compatibility constraints (9) is enforced. Again, $C_{\alpha}^{k}$ and $C_{\varsigma}^{k}$ restore the continuity of displacements at interfaces of mathematical layers. HWZZM is developed starting from the previous displacement field and similar to HWZZ inhibiting the decomposition into fictitious computational layers. Moreover, contributions to in-plane displacements over the third-order and to the transverse displacement over the fourth-order are neglected. As for HWZZ, membrane strains are derived from (14) with the decomposition into computational layers prevented, while it is allowed for deriving the expressions of out-of-plane strains. Membrane stresses $\sigma_{x x}, \sigma_{y y}, \sigma_{x y}$ are obtained from stress-strain relations, while out-of-plane master stresses come from integration of local equilibrium equations.

\subsection{Theories HWZZM(A-B-B2-C-C2-0)}

In this section, several theories are particularized from HWZZM by assuming a priori the expressions of some zigzag amplitudes, as specified below. Since contrary to what was done previously they are not determined through the imposition of physical and elasticity constraints, the theories of the present section resemble Murakami's like ones nowadays widely used, at least from the standpoint of the basic idea on which they are founded. Their purpose is that of highlighting that as physical constraints are only partially satisfied, in the present case due to the omission of certain interfacial stress compatibility conditions, it is no longer possible to obtain the same accuracy of ZZA, ZZA*, HWZZ and HWZZM with the same order of representation. On the contrary, it will be shown that displacement representation form and zig-zag functions can be assumed arbitrarily and the latter even omitted with no loss of accuracy, if instead the full set of constraints is enforced (see, section 4).

Amplitudes of HWZZMA are assumed coincident with those of HWZZM at the first interface from below, while $A_{k}^{u_{\varsigma}}(z)$ and $B_{k}^{u_{\varsigma}}(z)$ of HWZZMB are assumed the same of HWZZMA at the first interface from below, but $A_{k}^{u_{\alpha}}(z)$ is calculated like in HWZZM. In HWZZMC $B_{k}^{u_{\varsigma}}(z)$ is assumed uniform across the thickness (it is that by HWZZM at the first interface from below), while the remaining amplitudes are computed at each interface. In HWZZMO $B_{k}^{u_{\varsigma}}(z)$ are neglected, while $A_{k}^{u_{\alpha}}(z)$ and $A_{k}^{u_{\varsigma}}(z)$ are assumed the same of HWZZMB. HWZZMB2 and HWZZMC2 theories are similar to HWZZMB and HWZZMC, except amplitudes are those of HWZZM at the first interface from above.

Because out-of-plane stresses by these theories result discontinuous, integration of local equilibrium equations is required, with a corresponding increase in costs by $0.9 \%$. But at the same time, a savings of $10 \%$ is obtained, as zig-zag amplitudes are not computed at each interface, so in the end a positive is achieved which, however, is thwarted by the lack of accuracy.

\subsection{HWZZM* theory}

This theory is derived as a particularization of ZZA*, in order to assess the effect of omission of layerwise functions. It is developed in a similar way of HWZZ, omitting terms ${ }_{k} \tilde{c}^{i},{ }_{k} \tilde{C}_{\alpha}^{i}, \tilde{d}^{i}$ into displacement field (no decomposition into mathematical layers is allowed for displacement field):

$$
\begin{aligned}
& u_{\alpha}(x, y, z)=\left[u^{0}(x, y)+z\left(\Gamma_{\alpha}^{0}(x, y)-w^{0}(x, y)_{, \alpha}\right)\right]_{0}+\left\{\sum_{k=1}^{n_{\Im}}{ }_{k} \tilde{B}_{\alpha}^{i}(x, y) z\right\}_{c}+\left\{\left[C_{\alpha}^{i}(x, y) z^{2}\right]+\left[D_{\alpha}^{i}(x, y) z^{3}\right]\right\}_{i} \\
& u_{\varsigma}(x, y, z)=\left[w^{0}(x, y)\right]_{0}+\left\{\left[b^{i}(x, y) z\right]+\left[c^{i}(x, y) z^{2}\right]+\left[d^{i}(x, y) z^{3}\right]+e^{i}(x, y) z^{4}\right\}_{i}+\left\{\sum_{k=1}^{n_{\Im}}{ }_{k} \tilde{b}^{i}(x, y) z\right\}_{c}
\end{aligned}
$$

In this case, terms $[\ldots]_{i}$ only serve to satisfy stress-boundary conditions and local equilibrium equations, while $[\ldots]_{c}$ serve to satisfy stress compatibility conditions (9). Accordingly, the representation (15) is nothing but an equivalent form of (11). Regarding master strain field, contributions ${ }_{k} \tilde{C}_{\alpha}^{i}$ and ${ }_{k} \tilde{d}^{i}$ are restored, because decomposition into mathematical layers is allowed for calculation of out-of-plane strains. Finally, in-plane stresses are 
obtained from master strain field using stress-strain relations, while out-of-plane ones are obtained by integrating local equilibrium equations.

From a practical viewpoint, (15) imply a reduction of about $10 \%$ of the processing time per each layer with respect to ZZA and about of $6 \%$ with respect to HWZZ, but the goal is not so much this reduction, even if it becomes more important with the increase of the constituent layers, but instead that of proving how arbitrary layerwise functions give the same result once the full set of physical constrains $(6,8-10)$ is enforced.

\subsection{Lower order theories for comparisons}

Lower-order theories with some features reminiscent to ones of theories in the literature are particularized for sake of comparison. MHR considers piecewise cubic in-plane displacements incorporating Murakami's zig-zag function as the layerwise function, and a fourth-order polynomial transverse displacement:

$$
\begin{aligned}
& u_{\alpha}(x, y, z)=\left[u^{0}(x, y)+z\left(\Gamma_{x}^{0}(x, y)-w^{0}(x, y)_{, \alpha}\right)\right]_{0}+\left[C_{\alpha}(x, y) z^{2}+D_{\alpha}(x, y) z^{3}\right]_{i}+u_{\alpha z}(x, y) M^{k}(z) \\
& u_{\varsigma}(x, y, z)=\left[w^{0}(x, y)\right]_{0}+\left[a(x, y) z+b(x, y) z^{2}+c(x, y) z^{3}+d(x, y) z^{4}\right]_{i}
\end{aligned}
$$

where $M^{k}(z)$ is defined in section 3. Coefficients $C_{\alpha}, D_{\alpha}, a, b, c, d$ are calculated by enforcing the fulfilment of stress boundaries conditions (6), while $u_{\alpha z}$ is calculated by enforcing the fulfilment of first and second equilibrium equations (8) at the middle-plane of the laminate. The expressions of out-of-plane stresses are derived integrating local equilibrium equations, within the framework of HR variational theorem.

MHR \pm , is a variant where the right sign of Murakami's zig-zag function is determined at each interface on a physical basis (that which produces the least error in equilibrium equations), instead of being forced to reverse by the coefficient $(-1)^{k}$.

A refined variant MHR4 is obtained assuming the in-plane displacement of MHR and a fourth-order piecewise variation of the transverse displacement

$$
u_{\varsigma}(x, y, z)=\left[w^{0}(x, y)\right]_{0}+\left[a(x, y) z+b(x, y) z^{2}+c(x, y) z^{3}+d(x, y) z^{4}\right]_{i}+w_{z}(x, y) M^{k}(z)
$$

Coefficients $a$ to $d$ are determined by enforcing the fulfiment of stress boundary conditions (6), while $w_{z}$ is calculated by enforcing the fulfilment of the third local equilibrium equation at the middle-plane. Another theory, MHR4 \pm , is obtained by MHR4, assuming the right sign of Murakami's zig-zag functions at each interface on a physical basis, similarly to MHR土.

Theory MHWZZA has the same displacement-field of MHR (16), but its master stress field is that of HWZZ and, in addition, displacement, strain and stress fields are recovered using ZZA as post-processor.

Theory MHWZZA4 is derived assuming the in-plane displacement field by MHR, the transverse displacement by ZZA and as master strain and stress fields ones by HWZZ. So, the only substantial difference of MHWZZA4 with respect to HWZZ and ZZA is a different zig-zag function and a simplified kinematics, while MHR4, MHR4 \pm , MHR \pm and MHR are approximations obtained with increasing restrictions from MHWZZA4, in order to identify the effects played by single contributions omitted.

Theory HRZZ is developed within the framework of HR variational theorem postulating a uniform transverse displacement and a third-order zig-zag representation of in-plane displacements:

$$
\begin{aligned}
& u_{\alpha}(x, y, z)=\left[u^{0}(x, y)+z\left(\Gamma_{x}^{0}(x, y)-w^{0}(x, y)_{, \alpha}\right)\right]_{0}+\left[C_{\alpha}^{i}(x, y) z^{2}+D_{\alpha}^{i}(x, y) z^{3}\right]_{i}+\left[\sum_{k=1}^{n_{i}} \Phi_{\alpha}^{k}(x, y)\left(z-z_{k}\right) H_{k}(z)+\sum_{k=1}^{\Im}{ }_{\alpha} C_{u}^{k}(x, y) H_{k}\right]_{c} \\
& u_{\varsigma}(x, y, z)=w^{0}(x, y)
\end{aligned}
$$

then assuming the transverse normal stress $\sigma_{\varsigma \varsigma}$ the same of ZZA, while transverse shear stresses $\sigma_{\alpha \varsigma}$ are derived from equilibrium equations wherein membrane strains follows from (18). Because a uniform transverse displacement is chosen, transformed, reduced stiffness properties are assumed. 
Theory HRZZ4 assumes the same in-plane representation of HRZZ, the following fourth-order polynomial approximation of the transverse displacement:

$$
u_{\varsigma}(x, y, z)=\left[w^{0}(x, y)\right]_{0}+\left[b(x, y) z+c(x, y) z^{2}+d(x, y) z^{3}+e(x, y) z^{4}\right]_{i}
$$

and the same stress fields of HRZZ. In this case $\varepsilon_{\varsigma \varsigma}^{u}$ being no longer null it is not necessary to use transformed, reduced stiffness properties. Coefficients $b$ to $e$ are determined by enforcing the stress boundary conditions (6).

Regarding PP23 theory, in-plane displacements are parabolic, while the transverse one is cubic:

$$
\begin{aligned}
& u_{\alpha}(x, y, z)=\left[u_{\alpha}{ }^{0}(x, y)+z\left(\Gamma_{\alpha}^{0}(x, y)-w^{0}(x, y)_{, \alpha}\right)\right]_{0}+\left[\sum_{k=1}^{2} C_{k_{-} \alpha}^{i}(x, y) F_{k}^{\alpha}(z)+C_{\alpha}^{i}\right]_{i+c} \\
& u_{\varsigma}(x, y, z)=\left[w^{0}(x, y)\right]_{0}+\left[\sum_{k=1}^{3} D_{k}^{i}(x, y) G_{k}(z)+C_{\varsigma}^{i}\right]_{i+c} \\
& C_{k_{-} \alpha}^{i=1}=0 ; \quad C_{\alpha}^{i=1}=0 ; \quad C_{\varsigma}^{i=1}=0 ; \quad F^{\alpha}{ }_{k}(z)=G_{k}(z)=(z)^{k}
\end{aligned}
$$

Coefficients are calculated similarly to ZZA, by imposing the fulfilment of boundary conditions (6) and of continuity of out-of-plane stresses, of $\sigma_{\varsigma \varsigma, \varsigma}$ and of displacements (9)-(10) and the fulfilment of local equilibrium equations (8) at different points across the thickness. Results of out-of-plane stresses are post-processed by integrating local equilibrium equations.

Regarding ZZ theory, the displacement field is:

$$
\begin{aligned}
& u_{\alpha}(x, y, z)=\left[u_{\alpha}^{0}(x, y)+z\left(\Gamma_{\alpha}^{0}(x, y)-w^{0}(x, y)_{, \alpha}\right)\right]_{0}+C_{\alpha}(x, y) z^{2}+D_{\alpha}(x, y) z^{3}+\sum_{k=1}^{n_{i}} \Phi_{\alpha}^{k}(x, y)\left(z-z_{k}\right) H_{k}(z) \\
& u_{\varsigma}(x, y, z)=\left[w^{0}(x, y)\right]_{0}+\left[b(x, y) z+c(x, y) z^{2}+d(x, y) z^{3}+e(x, y) z^{4}\right]_{i}+\sum_{k=1}^{n_{i}} \Psi^{k}(x, y)\left(z-z_{k}\right) H_{k}(z)+\sum_{k=1}^{n_{i}} \Omega^{k}(x, y)\left(z-z_{k}\right)^{2} H_{k}(z)
\end{aligned}
$$

Coefficients $C_{\alpha}, D_{\alpha}, b, c, d, e$ are not redefined across the thickness and are obtained by imposing the boundary conditions (6) of out-of-plane stresses and of $\sigma_{\varsigma \varsigma, \varsigma}$, while $\Phi_{\alpha}^{k}, \Psi^{k}$ and $\Omega^{k}$ are calculated by imposing the continuity of transverse shear stresses and of its gradient (9) across the thickness. similarly to PP23 and other lower-order theories, out-of-plane stresses have to be post-processed and obtained by integrating local equilibrium equations.

\section{NEW THEORIES OF THIS PAPER}

New theories with a growing order of generalization are introduced below, which are aimed at demonstrating that the representation form of the displacement field and can be assumed arbitrarily and zig-zag functions can be omitted, whenever the full set of constraints $(6,8-10)$ is enforced and the coefficients of the representation are redefined across the thickness. The comparison with theories partially fulfilling constraints will show that instead the accuracy depends on the choices made, as known in literature for non-physically-based theories (see for example Catapano et al. (2011) and de Miguel et al. (2018)), which cannot fully satisfy $(6,8-10)$. Although zig-zag functions are not incorporated, the theories of this sections can still be considered as physically-based zig-zag theories, because constraints are enforced in order to determine the expressions of coefficients.

In spite displacements can be assumed arbitrarily, including a different representation for each component and from point to point across the thickness, unlike DL the d.o.f. remain fixed because coefficients, which are redefined through the use of symbolic calculations, aren't assumed as d.o.f. but instead they are fixed once for all, as outlined forward.

ZZA-X1 to ZZA-X4 new theories here presented derive from the parent theory ZZA-XX as particularizations obtained by specifying a different representations of the displacement field. ZZA-XX assumes the displacement field as an infinite series constituted by the product of initially unknown amplitudes and exponential functions of the thickness coordinate: 


$$
\begin{aligned}
& u_{\alpha}(x, y, z)=\left[\sum_{k=1}^{\infty} C_{k_{-} \alpha}^{i}(x, y) e^{\left(k z_{i} / h_{i}\right)}\right]_{\Im}+\sum_{j=1}^{\Im} D_{j_{-} \alpha}^{i}(x, y) \\
& u_{\varsigma}(x, y, z)=\left[\sum_{k=1}^{\infty} C_{k_{-} \varsigma}^{i}(x, y) e^{\left(k z_{i} / h_{i}\right)}\right]_{\Im}+\sum_{j=1}^{\Im} D_{j_{-} \varsigma}^{i}(x, y)
\end{aligned}
$$

Coefficients $C_{k_{-} \alpha}^{i}$ represent amplitudes that are recalculated across the thickness, hence contributions [ $]_{\Im}$ holds for each computational layer $\Im$. The expressions of $C_{k_{-} \alpha}^{i}, C_{k_{-} \zeta}^{i}, D_{j_{-} \alpha}^{i}$ and $D_{j_{-} \varsigma}^{i}$ are determined in the order they are listed by enforcing boundary conditions (6), compatibility conditions of out-of-plane stresses (9), the continuity of displacements across the thickness (10) and finally the fulfilment of local equilibrium equations at points across the thickness (8). Symbols $z_{i}$ and $h_{i}$ represent the thickness coordinate and the thickness of each layer, respectively, while $e^{\left(k z_{i} / h_{i}\right)}$ represent the primary functions, which will be specified differently in ZZA-X1 to ZZA-X4. However, note that the previous assignment of a fixed role to the coefficients is unnecessary since their role can be exchanged arbitrarily without accuracy suffering, as will be shown in the numerical applications subverting the purpose of coefficients, provided that the entire set of $(6,8-10)$ is enforced.

Actually coefficients $C_{k_{-} \alpha}^{i}, C_{k_{-} \zeta}^{i}$ assume the role of d.o.f., since to make the formulation more general, middle plane d.o.f. used so far in this paper are abandoned. To get as accurate results as ZZA the same number of physical constraints and conditions should be enforced in ZZA-XX, but obviously more other could be added to enrich accuracy, so it can be used in place of the exact solution if unavailable, as well as of 3-D FEA to estimate the accuracy of results of other cheaper theories.

A mutation of $Z Z A-X X$ here indicated as $Z Z A-X X^{\prime}$ is also considered, which is obtained by substituting the exponentials with powers:

$$
\begin{aligned}
& u_{\alpha}(x, y, z)=\left[\sum_{k=1}^{\infty} C_{k_{-} \alpha}^{i}(x, y) z^{(k)}\right]_{\Im}+\sum_{j=1}^{\Im} D_{j_{-} \alpha}^{i}(x, y) \\
& u_{\varsigma}(x, y, z)=\left[\sum_{k=1}^{\infty} C_{k_{-} \varsigma}^{i}(x, y) z^{(k)}\right]_{\Im}+\sum_{j=1}^{\Im} D_{j_{-} \varsigma}^{i}(x, y)
\end{aligned}
$$

in order to assess whether the representation form is immaterial. Naturally previous definitions of coefficients continues to be valid.

Four different particularizations ZZA-X1 to ZZA-X4 are introduced hereafter, whose representation is different for each displacement and varies across the thickness, so providing a much greater degree of generalization than all previous theories of this paper. For demonstration purposes, the displacement field of these theories is assumed differently from one another and differently for even and odd layers. It is assumed as the products of unknown amplitudes and a truncated series of general functions of z, indicated respectively as $F_{k}^{\alpha}$ and $G_{k}$, respectively for the in-plane and the transverse displacement components:

$$
\begin{aligned}
& u_{\alpha}(x, y, z)=\left[u_{\alpha}{ }^{0}(x, y)+z\left(\Gamma_{\alpha}^{0}(x, y)-w^{0}(x, y)_{, \alpha}\right)\right]_{0}+\left[\sum_{k=1}^{3} C_{k_{-} \alpha}^{i}(x, y) F_{\alpha}^{k}(z)+C_{\alpha}^{i}\right]_{i+c} \\
& u_{\varsigma}(x, y, z)=\left[w^{0}(x, y)\right]_{0}+\left[\sum_{k=1}^{4} D_{k}^{i}(x, y) G^{k}(z)+C_{\varsigma}^{i}\right]_{i+c}
\end{aligned}
$$

A different explicit definition of $F_{k}^{\alpha}$ and $G_{k}$ will define the different characteristics of ZZA-X1 to ZZA-X4. Differently from ZZA-XX here the d.o.f so far used (which still appear within the linear contributions $[\ldots]_{0}$ ) are reassumed. ZZA-X1 to ZZA-X4 are particularized as follows: 


$$
u_{\alpha} \triangleright{ }^{i} F_{\alpha}^{k}=\left\{\begin{array}{l}
z^{(k+1) / 2} \text { if } k=1,3 \\
e^{(k z /(2 h))} \text { if } k=2
\end{array}(k \max =3)\right\}
$$

even layers

$$
u_{\beta} \triangleright{ }^{i} F_{\beta}^{k}=\left\{z^{k} \quad(k=1,2,3)\right\}
$$

$$
u_{\varsigma} \triangleright{ }^{i} G^{k}=\left\{\begin{array}{l}
z^{(k+1) / 2} \text { if } k=1,3 \\
e^{(k z /(2 h))} \text { if } k=2,4
\end{array}(k \max =4)\right\}
$$

ZZA_X1

$$
u_{\alpha} \triangleright{ }^{i} F_{\alpha}^{k}=\left\{z^{k} \quad(k=1,2,3)\right\}
$$

odd layers

$$
\begin{aligned}
& u_{\beta} \triangleright{ }^{i} F_{\beta}^{k}=\left\{\begin{array}{l}
z^{(k+1) / 2} \text { if } k=1,3 \\
e^{(k z /(2 h))} \text { if } k=2
\end{array}(k \text { max }=3)\right\} \\
& u_{\varsigma} \triangleright{ }^{i} G^{k}=\left\{z^{k} \quad(k=1,2,3,4)\right\} \\
& u_{\alpha} \triangleright{ }^{i} F_{\alpha}^{k}=\left\{z^{k} \quad(k=1,2,3)\right\}
\end{aligned}
$$

even layers

$$
\begin{gathered}
u_{\beta} \triangleright{ }^{i} F_{\beta}^{k}=\left\{\begin{array}{l}
z^{(k+1) / 2} \text { if } k=1,3 \\
e^{(k z /(2 h))} \text { if } k=2
\end{array}(k \text { max }=3)\right\} \\
u_{\varsigma} \triangleright{ }^{i} G^{k}=\left\{\begin{array}{l}
z^{(k+1) / 2} \text { if } k=1,3 \\
e^{(k z /(2 h))} \text { if } k=2,4
\end{array}(k \text { max }=4)\right\}
\end{gathered}
$$

ZZA_X2

$$
u_{\alpha} \triangleright{ }^{i} F_{\alpha}^{k}=\left\{\begin{array}{l}
z^{(k+1) / 2} \text { if } k=1,3 \\
e^{(k z /(2 h))} \text { if } k=2
\end{array}(k \text { max }=3)\right\}
$$

odd layers

$$
\begin{gathered}
u_{\beta} \triangleright{ }^{i} F_{\beta}^{k}=\left\{\begin{array}{l}
z^{(k+1) / 2} \text { if } k=1,3 \\
e^{(k z /(2 h))} \text { if } k=2
\end{array}(k \text { max }=3)\right\} \\
u_{\varsigma} \triangleright{ }^{i} G^{k}=\left\{z^{k} \quad(k=1,2,3,4)\right\}
\end{gathered}
$$

$$
u_{\alpha} \triangleright{ }^{i} F_{\alpha}^{k}=\left\{\begin{array}{l}
z^{(k+1) / 2} \text { if } k=1,3 \\
e^{(k z /(2 h))} \text { if } k=2
\end{array}(k \text { max }=3)\right\}
$$

ZZA_X3

all layers

$$
\begin{aligned}
& u_{\beta} \triangleright{ }^{i} F_{\beta}^{k}=\left\{\begin{array}{l}
z^{(k+1) / 2} \text { if } k=1,3 \\
e^{(k z /(2 h))} \text { if } k=2
\end{array}(k \text { max }=3)\right\} \\
& u_{\varsigma} \triangleright{ }^{i} G^{k}=\left\{\begin{array}{l}
z^{(k+1) / 2} \text { if } k=1,3 \\
e^{(k z /(2 h))} \text { if } k=2,4
\end{array}(k \max =4)\right\} \\
& u_{\alpha} \triangleright{ }^{i} F_{\alpha}^{k}=\left\{z^{k} \quad(k=1,2,3)\right\}
\end{aligned}
$$

even layers

$$
\begin{gathered}
u_{\beta} \triangleright{ }^{i} F_{\beta}^{k}=\left\{\begin{array}{l}
z / h\left(\begin{array}{l}
z / h \\
\sin (z / h) \text { if } k=2 \\
e^{(k z /(2 h))} \text { if } k=3
\end{array}(k \max =3)\right.
\end{array}\right\} \\
u_{\varsigma} \triangleright{ }^{i} G^{k}=\left\{\begin{array}{l}
z^{k} i f k=1,3 \\
e^{(z / h)} \text { if } k=2,4
\end{array}(k \text { max }=4)\right\}
\end{gathered}
$$

ZZA_X4

$$
u_{\alpha} \triangleright{ }^{i} F_{\alpha}^{k}=\left\{\begin{array}{l}
z^{k} \text { if } k=1,3 \\
e^{(z / h)} \text { if } k=2
\end{array}(k \text { max }=3)\right\}
$$

odd layers

$$
\begin{gathered}
u_{\beta} \triangleright{ }^{i} F_{\beta}^{k}=\left\{\begin{array}{l}
z / h \quad \text { if } k=1 \\
\sin (z / h) \text { if } k=2 \\
e^{(k z /(2 h))} \text { if } k=3
\end{array}(k \text { max }=3)\right\} \\
u_{\varsigma} \triangleright{ }^{i} G^{k}= \begin{cases}z^{k} & (k=1,2,3,4)\}\end{cases}
\end{gathered}
$$


The previous forms of representation are chosen as examples that show that displacements can be arbitrarily assumed within the framework of physically based zig-zag theories of this paper, whenever conditions $(6,8-10)$ are enforced completely. In other words, the previous theories provide the same results of ZZA, ZZA*, HWZZM, HWZZM*, HWZZ, HSDT_34, ZZA*_43, ZZA-XX, ZZA-XX', ZZA_RDF, HWZZ_RDF, which are all united by the fact of satisfying the constraints just mentioned. In view of the arbitrariness of the representations assumed so far in (11)-(15), (20), (20') and considering further variants (24), (25), (28) discussed below will lead us to affirm that any form of representation can be assumed without any change in the results, if $(6,8-10)$ are enforced.

\subsection{ZZA_RDF}

This theory is developed assuming the same displacement field of ZZA, but a different role is attributed to coefficients in order to demonstrate that their function can be exchanged without the accuracy being affected:

$$
\begin{aligned}
& u_{\alpha}(x, y, z)=\left[u^{0}(x, y)+z\left(\Gamma_{\alpha}^{0}(x, y)-w^{0}(x, y)_{, \alpha}\right)\right]_{0}+\left[C_{\alpha}^{i}(x, y) z^{2}+D_{\alpha}^{i}(x, y) z^{3}\right]_{i}+\left[\sum_{k=1}^{n_{i}} \Phi_{\alpha}^{k}(x, y)\left(z-z_{k}\right) H_{k}(z)+\sum_{k=1}^{n_{\Im}} C_{u}^{k}(x, y) H_{k}(z)\right]_{c} \\
& u_{\varsigma}(x, y, z)=\left[w^{0}(x, y)\right]+\left[b^{i}(x, y) z+c^{i}(x, y) z^{2}+d^{i}(x, y) z^{3}+e^{i}(x, y) z^{4}\right]_{i}+\left[\sum_{k=1}^{n_{i}} \Psi^{k}(x, y)\left(z-z_{k}\right) H_{k}(z)+\sum_{k=1}^{n_{i}} \Omega^{k}(x, y)\left(z-z_{k}\right)^{2} H_{k}(z)+\sum_{k=1}^{n_{\Im}} C_{\varsigma}^{k}(x, y) H_{k}(z)\right]_{c}
\end{aligned}
$$

In the present case, terms $\Omega^{k}, \Psi^{k}, \Phi_{\alpha}^{k}$, are calculated by imposing equilibrium equations at different points across the thickness (for layers $\mathrm{i}>1$ ), while $C_{\alpha}^{i}, d^{i}$ and $e^{i}$ restore the continuity of out-of-plane stresses and of gradient of transverse normal stress across the thickness (for layers $i>1$ ). The numerical applications will show that results indistinguishable from those of ZZA and other theories derived from it that completely fulfill $(6,8-10)$ like the present theory, so demonstrating what claimed. It could be noticed that for some laminations in which one interface matches the middle reference plane, some stresses could be erroneously predicted to vanish for $z=0$; so, in this case apparently not every term can be used to impose compatibility stress conditions. Anyway, this issue can be overcome assuming a different reference plane; e.g., whose distance is $h_{d}>h / 2$ from the bottom face. In this case the displacement field can be rewritten as follows:

$$
\begin{aligned}
& u_{\alpha}(x, y, z)=\left[u^{0}(x, y)+\left(z-h_{d}+h / 2\right)\left(\Gamma_{\alpha}^{0}(x, y)-w^{0}(x, y)_{, \alpha}\right)\right]_{0}+\left[C_{\alpha}^{i}(x, y) z^{2}+D_{\alpha}^{i}(x, y) z^{3}\right]_{i}+\left[\sum_{k=1}^{n_{i}} \Phi_{\alpha}^{k}(x, y)\left(z-z_{k}\right) H_{k}(z)+\sum_{k=1}^{n_{3}}{ }_{\alpha} C_{u}^{k}(x, y) H_{k}(z)\right]_{c} \\
& u_{\varsigma}(x, y, z)=\left[w^{0}(x, y)\right]_{0}+\left[b^{i}(x, y) z+c^{i}(x, y) z^{2}+d^{i}(x, y) z^{3}+e^{i}(x, y) z^{4}\right]_{i}+\left[\sum_{k=1}^{n_{i}} \Psi^{k}(x, y)\left(z-z_{k}\right) H_{k}(z)+\sum_{k=1}^{n_{i}} \Omega^{k}(x, y)\left(z-z_{k}\right)^{2} H_{k}(z)+\sum_{k=1}^{n_{\text {S }}} C_{\varsigma}^{k}(x, y) H_{k}(z)\right]_{c} \\
& \left(h_{d} \leq z \leq h_{d}+h\right)
\end{aligned}
$$

whereas d.o.f. can still be referred to the middle plane of the laminate. Results will prove the results of $\left(24^{\prime}\right)$ to be identical to those provided by (24), so showing the choice of kinematics immaterial and the choice of the reference system unimportant when $(6,8-10)$ are enforced. Numerical tests conducted for verification purposes have also shown that the same results are obtained calculating $D_{\alpha}^{i}$ from the enforcement of the continuity of transverse shear stresses instead of calculating $C_{\alpha}^{i}$ as usually done, thus confirming the interchangeability of coefficients even with a different reference system.

\subsection{HWZZ_RDF}

This theory is developed as a variation of HWZZ wherein master displacement, strain and stress fields are the same of HWZZ, but $c^{i}$ terms (for $\mathrm{i}>1$ ) are calculated by imposing the continuity of the transverse normal stress gradient at the interfaces, while in HWZZ it was recovered through equilibria, instead of being explicitly imposed, otherwise small jumps of out-of-plane stresses take place as contributions $\Omega^{k}$ are omitted. Numerical results will show that contributions coming from HW variational principle that appear in HWZZ are unnecessary, as the same results can be achieved by HWZZ_RDF with a lower computational cost.

\subsection{ZZA*_43}

This theory is a modified version of ZZA*, whose in-plane displacements are constructed as a fourth-order piecewise polynomial, while the transverse one is piecewise cubic, that is, the expansion order is reversed: 


$$
\begin{aligned}
& u_{\alpha}(x, y, z)=\left[u^{0}(x, y)+z\left(\Gamma_{\alpha}^{0}(x, y)-w^{0}(x, y)_{, \alpha}\right)\right]_{0}+\left\{\sum_{k=1}^{n_{\Im}}{ }_{k} \tilde{B}_{\alpha}^{i}(x, y) z+\left[C_{\alpha}^{i}(x, y) z^{2}\right]+\left[D_{\alpha}^{i}(x, y) z^{3}+E_{\alpha}^{i}(x, y) z^{4}\right]+\sum_{k=1}^{n_{i}}{ }_{k} \tilde{C}_{\alpha}^{i}(x, y)\right\}_{i+c} \\
& u_{\varsigma}(x, y, z)=\left[w^{0}(x, y)\right]_{0}+\left\{\left[b^{i}(x, y) z+\sum_{k=1}^{n_{\Im}} \tilde{b}^{i}(x, y) z\right]+\left[c^{i}(x, y) z^{2}+\sum_{k=1}^{n_{i}}{ }_{k} \tilde{c}^{i}(x, y) z^{2}\right]+\left[d^{i}(x, y) z^{3}\right]+\sum_{k=1}^{n_{i}}{ }_{k} \tilde{d}^{i}(x, y)\right\}_{i+c}
\end{aligned}
$$

Similarly to ZZA*, terms ${ }_{k} \tilde{B}_{\alpha}^{i}, C_{\alpha}^{i},{ }_{k} \tilde{C}_{\alpha}^{i}$ serve the same purpose as $\Phi_{\alpha}^{k}, C_{\alpha}^{i}$ and ${ }_{\alpha} C_{u}^{k}$ of ZZA, while ${ }_{k} \tilde{b}^{i}$ and ${ }_{k} \tilde{c}^{i}$ have the function of $\Omega^{k}$ and $\Psi^{k}$ and ${ }_{k} \tilde{d}^{i}$ play the role of $C_{\varsigma}^{k}$. Once such coefficients are redefined across the thickness in order to satisfy physical constraints. Terms $C_{\alpha}^{i}, D_{\alpha}^{i}, E_{\alpha}^{i}, b^{i}, c^{i}$ and $d^{i}$ are calculated by imposing local equilibrium equations (8) and stress boundary conditions (9). Numerical results will show that the same results of ZZA and ZZA* $^{*}$ are obtained, so demonstrating that the expansion order of in-plane and out-of-plane components can be freely exchanged, provided that (6,8-10) are enforced. However it must be considered that the choice of position of equilibrium points across the thickness is very important for the present theory, as to get accurate results they have to be chosen near the interfaces, while in the case of ZZA they can be chosen within layers.

\subsection{HSDT_32, HSDT_33, HSDT_34}

Three enriched versions of HSDT are considered, whose in-plane displacements are still cubic, while the transverse one can be parabolic, cubic or of fourth order, and all differently to HSDT are of piecewise type because coefficients are recomputed layer-by-layer.

The purpose of these new theories is to demonstrate that redefinition of coefficients always improves accuracy of results and highlight which is the minimum expansion orders of in-plane and transverse displacements that still allow to get accurate results, both in terms of frequency and modal quantities.

They also help to prove that the representation can be chosen arbitrarily, as previously stated in (section 4.1). HSDT_32 theory has piecewise cubic in-plane displacements and a parabolic transverse one:

$$
\begin{aligned}
& u_{\alpha}(x, y, z)=\left[u^{0}(x, y)+z\left(\Gamma_{\alpha}^{0}(x, y)-w^{0}(x, y)_{, \alpha}\right)\right]_{0}+B_{\alpha}^{i}(x, y) z+C_{\alpha}^{i}(x, y) z^{2}+D_{\alpha}^{i}(x, y) z^{3}+E_{\alpha}^{i}(x, y) \\
& u_{\varsigma}(x, y, z)=\left[w^{0}(x, y)\right]_{0}+b^{i}(x, y) z+c^{i}(x, y) z^{2}+d^{i}(x, y)
\end{aligned}
$$

Coefficients $B_{\alpha}^{i}, C_{\alpha}^{i}, D_{\alpha}^{i}, b^{i}$ and $c^{i}$ are calculated by imposing out-of-plane stresses boundary conditions on transverse shear and normal stresses, their compatibility at interfaces and the fulfillment of local equilibrium equations across the thickness. Contributions $E_{\alpha}^{i}$ and $d^{i}$ enable the continuity of displacements, since the possibility of changing the representation across the thickness makes it necessary. The same holds for uniform contributions across the thickness of (27) and (28).

As discussed above in (section 4.3), it is not necessary to define the specific role of each one, because they can be freely exchanged.

Because conditions related to the gradient of the transverse normal stress cannot be imposed by (26), the following two more higher order theories HSDT_33, HSDT_34 are considered. HSDT_33 has a piecewise cubic polynomial transverse displacement:

$$
\begin{aligned}
& u_{\alpha}(x, y, z)=\left[u^{0}(x, y)+z\left(\Gamma_{\alpha}^{0}(x, y)-w^{0}(x, y)_{,}\right)\right]_{0}+B_{\alpha}^{i}(x, y) z+C_{\alpha}^{i}(x, y) z^{2}+D_{\alpha}^{i}(x, y) z^{3}+E_{\alpha}^{i}(x, y) \\
& u_{\varsigma}(x, y, z)=\left[w^{0}(x, y)\right]_{0}+b^{i}(x, y) z+c^{i}(x, y) z^{2}+d^{i}(x, y) z^{3}+e^{i}(x, y)
\end{aligned}
$$

Coefficients are still are calculated by imposing the full set of conditions $(6,8-10)$ but now less equilibrium points than for ZZA and for theories of sections 3.1, 3.2 and 4.1 to 4.3 equilibrium are sufficient because the number of terms is lower. Anyway results of this theory are better than those of HSDT_32.

Finally, theory HSDT_34 is developed, whose transverse displacement is a fourth-order piecewise polynomial:

$$
\begin{aligned}
& u_{\alpha}(x, y, z)=\left[u^{0}(x, y)+z\left(\Gamma_{\alpha}^{0}(x, y)-w^{0}(x, y)_{, \alpha}\right)\right]_{0}+B_{\alpha}^{i}(x, y) z+C_{\alpha}^{i}(x, y) z^{2}+D_{\alpha}^{i}(x, y) z^{3}+E_{\alpha}^{i}(x, y) \\
& u_{\varsigma}(x, y, z)=\left[w^{0}(x, y)\right]_{0}+b^{i}(x, y) z+c^{i}(x, y) z^{2}+d^{i}(x, y) z^{3}+e^{i}(x, y) z^{4}+f^{i}(x, y)
\end{aligned}
$$


This latter theory will be shown to be as accurate as the best former ones, so the fourth order expansion is the most appropriate choice for the transverse displacement, which so represents the necessary expansion order for getting the maximal accuracy.

\section{NUMERICAL ASSESSMENTS AND DISCUSSION}

The purpose of the present new theories is to confirm that previous findings of Icardi and Urraci (2018a, 2018b) ascertained on static cases still hold in dynamics, but above all the aim is to demonstrate that other new ones extending them still exist. As numerical applications that demonstrate the existence of such cases free vibrations of soft, flexible core sandwiches are presented, being well suited to highlight the different degree of accuracy of the theories considered to represent the layerwise effects involved, in particular those related to the transverse normal deformation which become dominant for pumping modes.

Numerical applications aim to show that: (i) the choice of zig-zag functions is immaterial; (ii) these functions can even be omitted from the displacement field (with a considerable advantage as regards the computational cost of the symbolic phase) once a sufficient number of coefficients is included in the displacement field that is recalculated at each interface in order to satisfy the constraints; (iii) the functions that represent the variation of displacements across the thickness can be arbitrarily chosen, depending on the component considered and the position across the thickness, provided that the full set of physical constraints $(6,8-10)$ is enforced, which justifies the appellation of physically-based given to the present theories. The aim of this study is also to show that: (iv) the redefinition of coefficients which is consequent to the imposition of constraints $(6,8-10)$ allows the theories of any order to significantly increase their accuracy; (v) exchanging order and form of representation of polynomial theories or the functions used in more general cases, results does not change if the full set of constraints $(6,8-10)$ is satisfied; (vi) certain lower-order theories with only a partial fulfillment of constraints can still be sufficiently accurate in predicting pumping modes, as well as modal through-thickness displacement and stress distributions; (vii) it is unnecessary to assign a specific role a priori to the displacement field coefficients, as they can be freely exchanged without the results change if $(6,8-10)$ are enforced and coefficients are redefined layer-by-layer. This study also has the intended aim of assessing which lower order theories only partially satisfying the constraints $(6,8-10)$, which as a consequence do not respect (i) to (iii) and so their accuracy depends on the choices made, are sufficient to adequately describe pumping motions.

In light of all this, it will be proven numerically that once the choice of zig-zag functions is immaterial, through the comparison of theories that assume these functions differently or omit them, when their coefficients are recomputed at each interface in order to satisfy the stress contact constraints. From the comparison of theories with a different representation of the displacement field it will be also shown that such the representation can be arbitrarily chosen and varied as desired across the thickness without results change. Comparisons will also show that the redefinition of coefficients, which significantly increase accuracy, also makes immaterial to assign a specific role a priori to the coefficients, as the results don't change. Lower-order theories will be shown sufficiently accurate in predicting pumping modes, as well as modal through-thickness displacement and stress distributions in a number of cases but not always, which instead applies for higher-order counterparts. As regards the aspects related to the theoretical modelling of natural frequencies and relative through-thickness modal distributions of stress and displacements, the detailed aspects coming from the analysis of the results of the individual cases shows the following discussed in the subsequent sessions.

\subsection{A premise about terminology}

Note that in the discussion that follows and throughout the paper the appellation of higher-order theories is reserved to ZZA Icardi and Sola (2014), ZZA*, HWZZM, HWZZM* retaken from Icardi and Urraci (2018a), HWZZ Icardi and Urraci (2018b), HSDT_34, ZZA*_43, ZZA-XX, ZZA-XX', ZZA_RDF, HWZZ_RDF and ZZA_X1, to _X4 introduced in this paper, while the appellation lower-order is attributed to theories HWZZMA, B, B2, C, C2 and 0 and MHR MHR4 \pm retaken from Icardi and Urraci (2018a), HRZZ, HRZZ4, MHWZZA, MHWZZA4, MHR, MHR4 retaken from Icardi and Urraci (2018b), PP23, ZZ retaken from Icardi (2001) and HSDT_32, HSD_33 introduced in this paper, in addition to FSDT (shear correction factor 5/6) and HSDT used for comparisons. It is specified that FSDT is derived from (4) including only the contribution $[\ldots]_{0}$, while the second adds also a cubic contribution thanks to which shear stress free boundary conditions can be satisfied. With regard HSDT and FSDT theories, it should also be specified that results previously given in Icardi and Urraci (2018a) have been computed assuming simply-supported edges applied on the middle reference plane $\Omega$, while in the present paper they are applied to the whole cross section, as allowed by the three- 
dimensional model ZZA from which HSDT is particularized. It should be noted that eigen-frequencies computed with the constraints considered in Icardi and Urraci (2018a) by HSDT considerably differ from the present ones, which result more similar to those in the literature being much lower, while FSDT is indifferent to where the boundary conditions are placed, due to its extremely simplified kinematics.

\subsection{A Discussion of cases examined}

The accuracy of previous theories in predicting free vibration modes of flexible soft-core sandwich beams and plates is assessed considering mainly thick structures for which layerwise effects take on the utmost importance. However, the effect of increasing the length-to-thickness ratio and of varying the orthotropy ratio in one case is also investigated using the various theories developed in this paper to confirm the already well known results in the literature. Comparisons will be carried out with 3-D FEA (Icardi and Atzori (2004)), exact and reference solution retaken from literature. Lay-up, material properties of constituent layers, dimensions, boundary conditions, normalizations, trial functions and expansion order used for each case are given in Tables 2 and 4.

\subsection{Case a}

It constitutes a preliminary test that is considered in order to check the correct implementation of the theories of this paper and simultaneously evaluate how errors of lower-order ones grow varying the length-to-thickness and orthotropy ratios. The first three natural frequencies of a 0-90-0 laminate are considered and compared with the first frequency provided by Kim (2007) (see, Table 5). Results are reported varying the length-to-thickness ratio from 4, to 10 , to 20 and the orthotropy ratio $E_{1} / E_{2}$ from 3 , to 25 , to 40 .

Table 4 Mechanical Properties. Material m, E1/E2=3, 25, $40(=m 1, m 2, m 3)$, where E2=6.89GPa. Material $n$ : the following orthotropic stiffness properties are assumed: Q11 $=10^{5} \mathrm{MPa} ; \mathrm{Q} 12=0.2331{ }^{*} \mathrm{Q} 11 ; \mathrm{Q} 13=0.010776 * \mathrm{Q} 11 ; \mathrm{Q} 22=0.543103 * \mathrm{Q} 11$; $\mathrm{Q} 23=0.098276 * \mathrm{Q} 11 ; \mathrm{Q} 33=0.530172 * \mathrm{Q} 11 ; \mathrm{Q} 55=0.26681 * \mathrm{Q} 11 ; \mathrm{Q} 44=0.159914 * \mathrm{Q} 11 ; \mathrm{Q} 66=0.262931 * \mathrm{Q} 11 ; \mathrm{density}=1627 \mathrm{~kg} / \mathrm{m}^{3}$.

\begin{tabular}{|c|c|c|c|c|c|c|c|c|c|c|}
\hline $\begin{array}{l}\text { Material } \\
\text { name }\end{array}$ & $\begin{array}{c}\text { E1 } \\
{[\mathrm{GPa}]}\end{array}$ & $\begin{array}{c}\text { E2 } \\
\text { [GPa] }\end{array}$ & $\begin{array}{c}\text { E3 } \\
\text { [GPa] }\end{array}$ & $\begin{array}{c}\text { G12 } \\
\text { [GPa] }\end{array}$ & $\begin{array}{c}\text { G13 } \\
\text { [GPa] }\end{array}$ & $\begin{array}{c}\text { G23 } \\
\text { [GPa] }\end{array}$ & u12 & u13 & $u 23$ & $\begin{array}{c}\rho \\
{[\mathrm{kg} / \mathrm{m} 3]}\end{array}$ \\
\hline$m$ & $\overline{E 1}$ & $\overline{E 2}$ & $\overline{E 2}$ & $0.5 \mathrm{E} 2$ & $0.5 \mathrm{E} 2$ & $0.2 \mathrm{E} 2$ & 0.25 & 0.25 & 0.25 & 1558.35 \\
\hline o1 & 33.5 & 8 & 8 & 2.26 & 2.26 & 3 & 0.35 & 0.35 & 0.35 & 1627 \\
\hline 02 & 139 & 3.475 & 3.475 & 1.7375 & 1.7375 & 0.695 & 0.25 & 0.25 & 0.25 & 1627 \\
\hline$p$ & 6.89 & 6.89 & 6.89 & 3.45 & 3.45 & 3.45 & 0 & 0 & 0 & 97 \\
\hline$q$ & 0.035 & 0.035 & 0.035 & 0.0123 & 0.0123 & 0.0123 & 0.4 & 0.4 & 0.4 & 32 \\
\hline $\mathrm{r} 1$ & 36.23 & 10.62 & 7.21 & 5.6 & 5.68 & 3.46 & 0.26 & 0.33 & 0.48 & 1800 \\
\hline r2 & 190 & 7.7 & 7.7 & 4.2 & 4.2 & 2.96 & 0.3 & 0.3 & 0.3 & 1600 \\
\hline s & 0.036 & 0.036 & 0.036 & 0.013 & 0.013 & 0.013 & 0.38 & 0.38 & 0.38 & 32 \\
\hline $\mathrm{t}$ & 0.070 & 0.070 & 0.070 & 0.019 & 0.019 & 0.019 & 0.3 & 0.3 & 0.3 & 52.1 \\
\hline u & 0.020 & 0.020 & 0.020 & 0.012 & 0.012 & 0.012 & 0.3 & 0.3 & 0.3 & 39.7 \\
\hline
\end{tabular}

No modal distributions of stresses and displacements are given for this case since it does not represent a particularly challenging benchmark. Indeed, most theories, i.e. excluded some of the lowest-order ones, provide accurate results, both as regards the frequencies and the modal distributions of displacement and stresses across the thickness, which in any case are already well known cross-ply laminates being extensively studied, as shown in the literature. Anyway, although this benchmark is not particularly difficult, it is still interesting because it is widely studied in the literature, so it allows a direct comparisons with the results of other researchers. It is interesting also why on a closer inspection the existence of small discrepancies are shown, that will become much more evident in the next cases, how it transpires from the results of this paper. So this case, already allows to start identifying the different behavior of structural models due to their different assumptions although the material differences come from the sole orientation difference of the sheets. The results of Table 5 confirm the well-known role according to which as the length / thickness ratio decreases, the ratio of orthotropy increases and going towards the higher modes, the discrepancies between the theories increase. It is also confirmed that for laminates consisting of identical re-oriented sheets it is not very important to accurately describe the normal transverse deformation, even if it turns out that the theories more accurately describing this effect are slightly more accurate. 
Table 5 Normalized frequencies, case a.

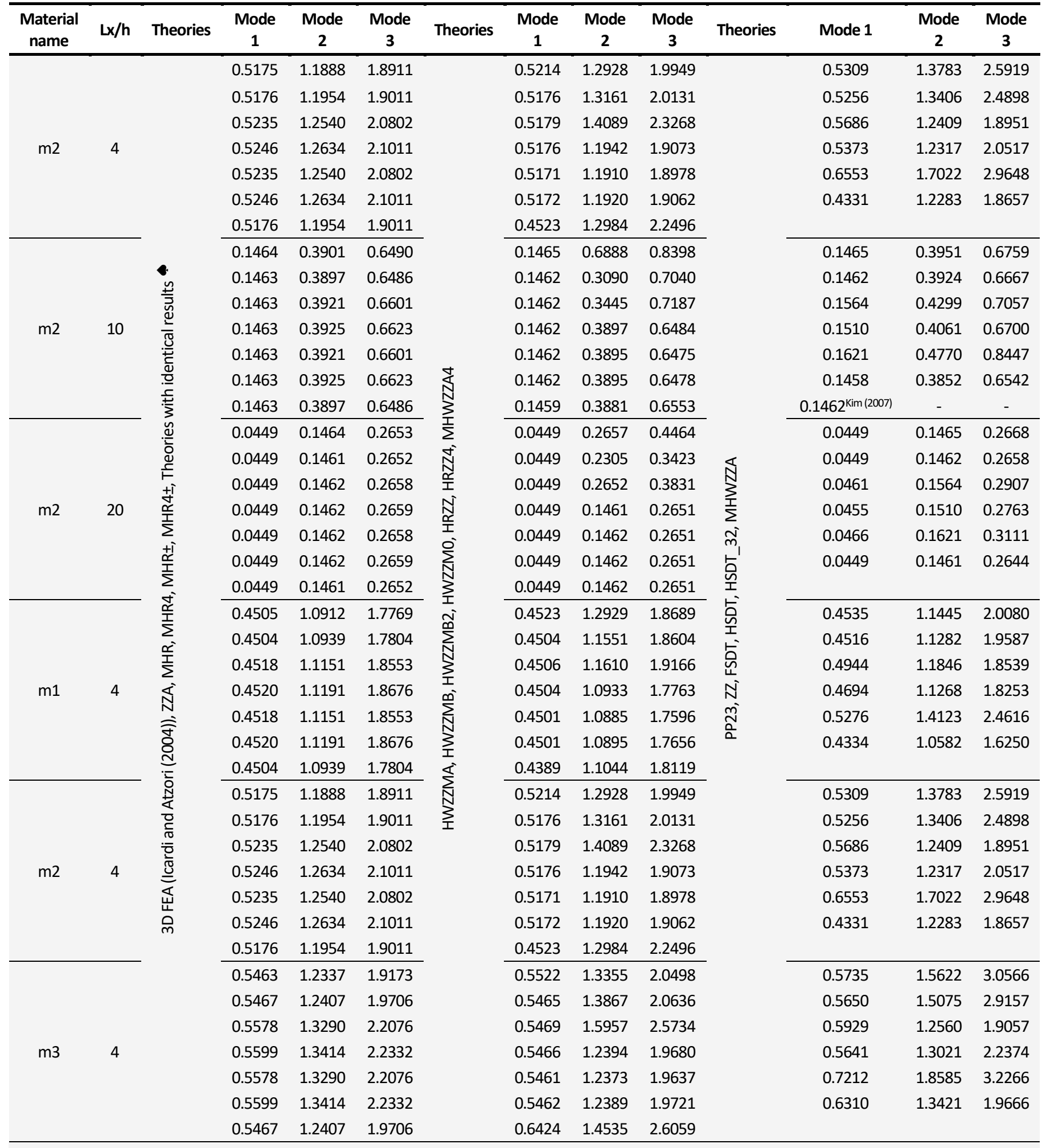

• ZZA*, HWZZ, HWZZM, HWZZM*, HWZZMC, HWZZMC2, HSDT_33, HSDT_34, ZZA*_43, ZZA_RDF, HWZZ_RDF, ZZA_X1, ZZA_X2, ZZA_X3, ZZA_X4, ZZA-XX, ZZA-XX' (error < 1\%); Modes with 1,2,3 halfwaves

\subsection{Case b}

An additional preliminary test is retaken from Cho et al. (1991), in order to further assess the correct implementation of theories and begin to distinguish their degree of accuracy, as a result of their assumptions. 
Although not yet being a particularly challenging benchmark for theories, the present case is an interesting test case because it regards a monolayer for which pumping modes occurs as the first modes, as a consequence of its material properties (see Table 4).

It should be noted that being a single-layer structure it masks any errors inherent to an imperfect description of stress-continuity effects (9) which precisely presuppose the existence of interfaces between different materials that do not exist here. Nevertheless, it represents an interesting case because it highlights just the ability of theories to describe the transverse normal deformation. Consequently, it offers the possibility of testing the theories as far as the ability to adequately describe normal transverse deformation is concerned. However, it is not yet a very challenging and particularly suited case since its effects related to the transverse normal deformability are still rather mild, beyond the fact that pumping starts to occur. Anyway, the present is a case that allows to gradually discriminate the accuracy of theories in later stages up to more difficult cases from the point of view of modeling presented next.

Being a monolayer and therefore not producing layerwise effects, it is not suitable for discriminating the theories. It could erroneously make us assume valid theories that are not appropriate for much difficult cases. In fact, even polynomial theories that will appear unsuitable for the next more challenging cases seem to be accurate in the present case, which so provides deceptive indications.

Nevertheless to this and although it does not appear to be particularly selective, this case has often been considered by researchers who have developed sandwich theories, so becoming a standard test case and, consequently, comparative results can be found in the literature. The available results, as well as the present ones of Table 6, show that some modes are asymmetric while others are symmetrical with respect to the middle plane and that almost all the theories considered in this paper are adequately accurate.

Table 6 Normalized frequencies, case b.

\begin{tabular}{|c|c|c|c|c|c|c|c|c|c|c|c|c|c|}
\hline \multirow[t]{2}{*}{ Theories } & \multicolumn{5}{|c|}{ Mode with $(n, m)$ waves } & \multirow[t]{2}{*}{$\begin{array}{l}(n, m) \\
\text { waves }\end{array}$} & \multirow[t]{2}{*}{ Theories } & \multicolumn{5}{|c|}{ Mode with $(n, m)$ waves } & \multirow[t]{2}{*}{$\begin{array}{l}(n, m) \\
\text { waves }\end{array}$} \\
\hline & 0.0474 & 0.2170 & 0.3941 & 1.3077 & 1.6530 & & & 0.1033 & 0.3450 & 0.5624 & 1.3331 & 1.7160 & \\
\hline \multirow{15}{*}{ 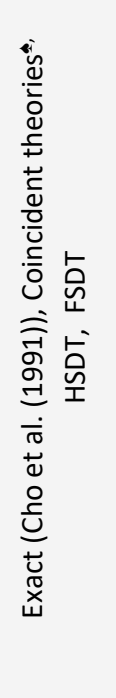 } & 0.0474 & 0.2169 & 0.3940 & 1.3085 & 1.6543 & $(1,1)$ & \multirow{11}{*}{ 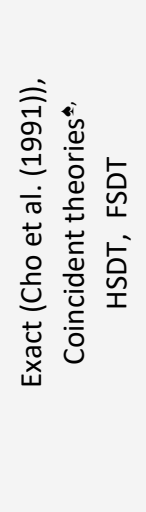 } & 0.1033 & 0.3450 & 0.5624 & 1.3339 & 1.7184 & $(1,2)$ \\
\hline & 0.0474 & - & - & 1.3086 & 1.6549 & & & 0.1031 & - & - & 1.3339 & 1.7208 & \\
\hline & 0.0473 & - & - & 1.3078 & 1.6540 & & & 0.1031 & - & - & 1.3331 & 1.7201 & \\
\hline & 0.1188 & 0.3515 & 0.6728 & 1.4205 & 1.6805 & \multirow{4}{*}{$(2,1)$} & & 0.1694 & 0.4338 & 0.7880 & 1.4316 & 1.7509 & \multirow{4}{*}{$(2,2)$} \\
\hline & 0.1188 & 0.3515 & 0.6728 & 1.4215 & 1.6819 & & & 0.1694 & 0.4338 & 0.7880 & 1.4324 & 1.7535 & \\
\hline & 0.1187 & - & - & 1.4215 & 1.6826 & & & 0.1692 & - & - & 1.4323 & 1.7560 & \\
\hline & 0.1185 & - & - & 1.4209 & 1.6817 & & & 0.1698 & - & - & 1.4316 & 1.7554 & \\
\hline & 0.1888 & 0.4953 & 0.7600 & 1.3765 & 1.8115 & \multirow{4}{*}{$(1,3)$} & & 0.2180 & 0.5029 & 0.9728 & 1.5778 & 1.7334 & \multirow{4}{*}{$(3,1)$} \\
\hline & 0.1888 & 0.4953 & 0.7601 & 1.3772 & 1.8156 & & & 0.2180 & 0.5029 & 0.9728 & 1.5788 & 1.7351 & \\
\hline & 0.1884 & - & - & 1.3772 & 1.8207 & & & 0.2180 & - & - & 1.5788 & 1.7360 & \\
\hline & 0.1881 & - & - & 1.3764 & 1.8203 & & & 0.2172 & - & - & 1.5782 & 1.7353 & \\
\hline & 0.3320 & 0.6504 & 1.1814 & 1.5737 & 1.9289 & \multirow{4}{*}{$(3,3)$} & & & & & & & \\
\hline & 0.3321 & 0.6504 & 1.1816 & 1.5744 & 1.9338 & & & & & & & & \\
\hline & 0.3315 & - & - & 1.5744 & 1.9390 & & & & & & & & \\
\hline & 0.3302 & - & - & 1.5736 & 1.9388 & & & & & & & & \\
\hline
\end{tabular}

This is due to the secondary importance of transverse normal deformation effect, because flexural modes characterized by a though-thickness symmetric transverse displacement with a magnitude of an order lower than the in-plane components, which on the contrary are antisymmetric. A similar behavior is highlighted as regards the magnitude of $\sigma_{\varsigma \varsigma}$ compared to that of $\sigma_{\alpha \varsigma}$ and $\sigma_{\beta \varsigma}$, but the difference becomes of several orders of magnitude less.

Instead the modes attributable as pumping show an antisymmetric $u_{\varsigma}$ and symmetrical $u_{\alpha}, u_{\beta}$ with the same amplitude ratios as before, while $\sigma_{\varsigma \varsigma}$ continues to be several orders of magnitude less. For this case, the modes attributable as flexural are those at lower frequencies, while those identified as pumping modes occur subsequently.

As far as the theories MHR, MHR4, MHR \pm , MHR4 \pm , MHWZZA, MHWZZA4 are concerned, it should be noted that more accurate results are obtained for this monolayer case by deleting zig-zag functions, because with the choices made they do not automatically vanish (errors up to $5 \%$ on frequencies) as on the contrary it rightly occurs for physically-based ones. As there is not much dispersion of the results of the theories, figures relating to the modal distributions of displacements and stresses across the thickness are not presented for this case. 


\subsection{Cases $\mathrm{c}$ to $\mathrm{g}$}

Challenging cases not yet proposed in the literature are considered hereafter to prove what claimed at the beginning of section 5 regarding the choice of zig-zag and representation functions. Moreover, due to their characteristics, these cases also lend themselves to show that theories with an enriched representation across the core and considering the constituent individual laminae rather than a single sheet forming the face, should be chosen to capture pumping and other effects that trigger other 3-D phenomena and so not overlooking them as will be shown by the present numerical results.

These latter considerations, which are in conflict with what still often proposed in literature, namely that it is sufficient to carry out the analysis of sandwiches three-layer sandwich theories instead of using layerwise theories, constitutes the further purpose of numerical tests. It results in highlighting that layerwise theories are unnecessary unless not demanding cases with mild variations of displacements and stresses across the interfaces of core are considered, like the previous ones, while for those from here on out their use is advisable.

It is emphasized that hereafter cases involving properties commonly used by the industry are considered and not ad hoc constructed cases just in order to justify theories that otherwise would have little practical use. So ultimately this paper claims through the numerical results of these last sections that it is essential to provide a very accurate description of layerwise effects, but discrete-layer models are unnecessary for doing so, most of the present theories having a fixed number of variables and thus being cost-effective being accurate. The last purpose prefixed is to understand how far it is necessary to go when considering refined theories for capturing pumping modes. In light of the above considerations, a fairly wide and diversified number of cases are discussed hereafter which lend to highlight the relative merits of theories, some of which reproduces characteristics similar to those of theories already published.

Extremely thick cases, which apparently contradict the intent of considering only materials of industrial interest that are commonly used, are studied here because they better highlight the different degree of accuracy offered by theories, given their very strong layerwise effects, and therefore allow to better understand their intrinsic characteristics.

Case c, which is retaken from Icardi and Urraci (2018a), concerns a sandwich plate with elastic and thickness properties of faces and core commonly used in the industrial applications. So, unlike the previous cases, variations of more than one order of magnitude of elastic coefficients and a distinctly different thickness of constituent layers are considered. Specifically, the core is thick and has rather weak properties compared to laminated faces (Table 4) that enhance layerwise effects

Table 7 Normalized frequencies, case c.

\begin{tabular}{|c|c|c|c|c|c|c|}
\hline THEORY & $\begin{array}{c}\text { Mode } 1 \\
(1,1)\end{array}$ & $\begin{array}{c}\text { Mode } 2 \\
(2,1)\end{array}$ & $\begin{array}{c}\text { Mode } 3 \\
(1,2)\end{array}$ & $\begin{array}{c}\text { Mode } 4 \\
(2,2)\end{array}$ & $\begin{array}{c}\text { Mode } 5 \\
(3,1)\end{array}$ & $\begin{array}{c}\text { Mode } 6 \\
(1,1)\end{array}$ \\
\hline 3D FEA Icardi and Atzori (2004) & 1.6882 & 2.8796 & 3.4723 & 4.3033 & 4.6899 & 5.7441 \\
\hline Theories with identical results & 1.6898 & 2.8855 & 3.4777 & 4.3171 & 4.7030 & 5.7500 \\
\hline HWZZMA & 5.3141 & 7.2110 & 11.3333 & 19.3264 & 21.7117 & 46.2461 \\
\hline HWZZMB & 1.7010 & 2.8913 & 3.5605 & 4.3693 & 4.7101 & 34.1888 \\
\hline HWZZMB2 & 1.7651 & 4.7210 & 3.5473 & 5.0578 & 10.6544 & 34.7002 \\
\hline HWZZMO & 2.5169 & 5.6364 & 5.9019 & 8.8827 & 11.3106 & 34.3120 \\
\hline HRZZ & 1.6823 & 2.8517 & 3.3940 & 4.1648 & 4.5907 & 34.3046 \\
\hline HRZZ4 & 1.6821 & 2.8525 & 3.3965 & 4.1720 & 4.5948 & 34.1832 \\
\hline MHWZZA & 11.7654 & 2.7153 & 2.7264 & 3.7526 & 6.8737 & 1.4635 \\
\hline MHWZZA4 & 1.1776 & 3.9325 & 4.3165 & 4.3950 & 4.5656 & 5.6519 \\
\hline MHR & 12.7147 & 15.1380 & 16.4288 & 27.1626 & 27.6009 & 64.6322 \\
\hline MHR4 & 12.7626 & 16.6121 & 22.2689 & 27.7771 & 27.8687 & 75.2673 \\
\hline MHR \pm & 1.6959 & 2.9097 & 3.4919 & 4.3405 & 4.7643 & 61.7387 \\
\hline MHR4 \pm & 5.1510 & 5.8356 & 6.7704 & 7.2618 & 7.2672 & 66.5689 \\
\hline PP23 & 1.7180 & 3.0361 & 3.6828 & 4.6462 & 5.1672 & 7.4814 \\
\hline $\mathrm{ZZ}$ & 1.6914 & 2.9610 & 3.4900 & 4.3536 & 5.0224 & 5.8191 \\
\hline HSDT_32 & 1.7083 & 3.7725 & 5.5851 & 6.5974 & 6.8196 & 7.6773 \\
\hline HSDT Icardi and Urraci (2018a) & 16.5610 & 28.7206 & 37.7283 & 44.0301 & 44.2319 & - \\
\hline HSDT & 3.9263 & 5.9589 & 6.8677 & 8.2366 & 8.4810 & - \\
\hline FSDT & 11.0783 & 17.6361 & 20.9784 & 25.0619 & 25.3697 & - \\
\hline \multicolumn{7}{|c|}{$\begin{array}{c}\text { ZZZA, ZZA*, HWZZ, HWZZM, HWZZM*, HWZZMC, HWZZMC2, HSDT_33, HSDT_34, ZZA*_43, ZZA_RDF, HWZZ_RDF, ZZA_X1, ZZA_X2, } \\
\text { ZZA_X3, ZZA_X4, ZZA-XX, ZZA-XX' (error < } 1 \%) ;\end{array}$} \\
\hline
\end{tabular}


This case is considered in order to discern the different behavior of theories, because like for all sandwiches with similar characteristics, the strong variations of properties of constituent layers enhances the effects of the different assumptions made as regards the description of transverse shear and normal deformations largely influencing pumping motions.

The results of Table 7 show that, due to the aforementioned strong differences between the material properties of faces and core, there is a greater dispersion between the theories than in the previous cases. Some lower-order theories, (specifically MHR, MHR4, MHR4 , MHWZZA, MHWZZA4, HWZZMA, HWZZMB, HWZZMB2, HWZZMO, FSDT and HSDT) are no longer accurate as in the previous two cases. Others still accurately predict the flexural frequencies but are unable to represent the pumping modes because of their bad description of the normal transverse deformation (HRZZ, HRZZ4, MHR \pm ). Remaining lower-order theories inaccurately predict the modal distributions of displacements and stresses, but instead quite accurately predict the frequencies. It could be noticed that theories misestimating frequencies, i.e. lower-order ones, predict modal displacements with trends that go against those of more accurate theories as regards the in-plane components, while less big errors are made for the transversal component. In-plane stresses of less accurate theories already show discrepancies with respect to the other theories, but the largest discrepancies are shown for out-of-plane components, a sign of the lesser ability of lower-order theories to describe 3-D effects.

As an example in Figure 1 the through-thickness variation of $\sigma_{\alpha \varsigma}$ for mode 1 is reported for all theories. It is noted that HRZZ4 and MHWZZA4 underestimate such stress across the core and also across the faces, while MHWZZA, MHR and MHR4 overestimate it, while FSDT and HSDT are not able to provide realistic results even when the values obtained by integration from equilibrium equations are reported, which confirms what is already known in the literature. Similar considerations apply to the other two out-of-plane stress components.

As shown in Table 7, only the highest-order theories ZZA, ZZA*, HWZZM, HWZZM*, HWZZ, HSDT_34, ZZXX_43, ZZAXX, ZZAXX', ZZA_RDF, HWZZ_RDF and ZZA_X1 to X4 accurately predict pumping modes, but it is noted however by results not reported for brevity that even HSDT_33 and ZZ are able to adequately predict frequencies and modal distributions.

Theory HSDT_34 is accurate and efficient, while its counterparts HSDT_32, HSDT_33 appear to be much less so. All this demonstrates that only an accurate piecewise description of the transverse displacement, like those of HSDT_34 and other higher-order theories, is adequate for this case.
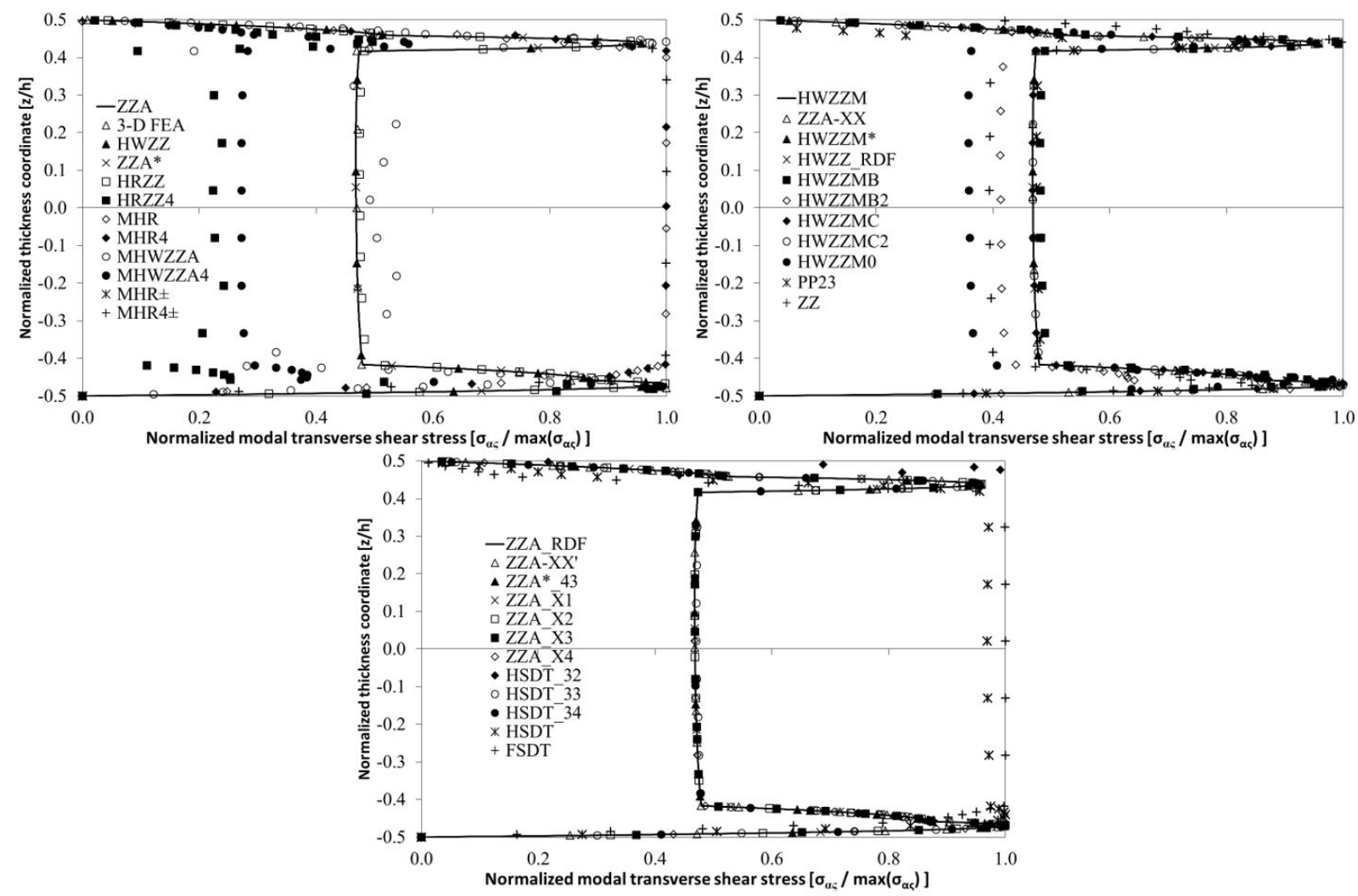

Figure 1 Case c: Normalized modal transverse shear stress (mode 1, bending). 
The behavior of each lower-order theory being strongly case-dependent, as emerges from the previous considerations and from ones of cases above, it is not possible to deduce rules of general character, but instead they must limit to the single case. Considering the fact that the computational cost of all higher-order theories of this paper, which are always the most accurate ones, is still comparable with that of lower-order counterparties (see Table 3) which are not always accurate, the latter do not bring any advantage and therefore become uninteresting.

The results of this case demonstrate the validity of what claimed, that the choice of zig-zag functions is immaterial, these functions can even be omitted from the displacement field. The functions that represent the variation of displacements across the thickness can be arbitrarily chosen, the redefinition of coefficients allows the theories of any order to significantly increase their accuracy, exchanging order and form of representation results does not change and that certain lower-order theories with only a partial fulfillment of constraints can still be sufficiently accurate. Moreover, it is unnecessary to assign a specific role a priori to the displacement field coefficients, as they can be freely exchanged without the results change.

Case $\mathbf{d}$. This case, which is obtained from previous one varying the material properties of core, aims to highlights that vibration modes are strongly influenced by modest changes in properties. In particular, pumping modes can disappear from first frequencies with even small changes, so it is not easy to decide a priori if to use higher or lowerorder models, since it is not easy to guess whether the transverse normal deformation has a primary role or not. However, considering that the higher-order theories are remarkably efficient in reality, the problem does not arise. Although a no easily identifiable general rule can be drawn from the results presented in this paper, it will be cleared from the results presented in Table 8 for this case, that higher-order theories of this paper can be applied in all cases without weighing negatively on costs (Table 3 ). So it is not necessary to operate a priori any potentially risky choice for the accuracy of the results opting for any of lower order theories. Furthermore, it can be noticed from Table 3 that lower-order theories are not most economically convenient compared to higher-order counterparts because postprocessing operations are necessary to achieve the necessary degree of accuracy.

It remains to examine the details regarding the various theories considered, that the numerical results highlight. For this case similar considerations apply to those of case c, but now there are more dispersions of the results (especially for mode 4).

Table 8 Normalized frequencies, case d.

\begin{tabular}{|c|c|c|c|c|c|c|}
\hline THEORY & $\begin{array}{c}\text { Mode } 1 \\
(1,1)\end{array}$ & $\begin{array}{c}\text { Mode } 2 \\
(2,1)\end{array}$ & $\begin{array}{c}\text { Mode } 3 \\
(1,2)\end{array}$ & $\begin{array}{c}\text { Mode } 4 \\
(2,2)\end{array}$ & $\begin{array}{c}\text { Mode } 5 \\
(3,1)\end{array}$ & $\begin{array}{c}\text { Mode } 6 \\
(3,2)\end{array}$ \\
\hline 3D FEA Icardi and Atzori (2004) & 3.1542 & 5.1028 & 5.5209 & 6.8629 & 7.5472 & 8.8919 \\
\hline Theories with identical results & 3.1633 & 5.1395 & 5.5511 & 6.9273 & 7.6341 & 9.0113 \\
\hline HWZZMA & 6.5590 & 8.9323 & 14.0140 & 27.4411 & 27.5244 & 28.4907 \\
\hline HWZZMB & 3.1691 & 5.1555 & 5.6482 & 6.9579 & 7.7820 & 9.0647 \\
\hline HWZZMB2 & 3.2500 & 5.7445 & 5.9271 & 7.7215 & 10.5917 & 11.2177 \\
\hline HWZZMO & 3.6985 & 7.1062 & 7.5328 & 10.5826 & 12.8762 & 16.1719 \\
\hline HRZZ & 3.1479 & 5.0779 & 5.4561 & 6.7539 & 7.4598 & 8.6694 \\
\hline HRZZ4 & 3.1514 & 5.0913 & 5.4767 & 6.7941 & 7.4976 & 8.7509 \\
\hline MHWZZA & 1.3295 & 2.3539 & 2.9193 & 3.1509 & 3.5257 & 8.2946 \\
\hline MHWZZA4 & 1.3279 & 3.6122 & 3.7515 & 3.8117 & 4.1134 & 5.4391 \\
\hline MHR & 13.8886 & 16.7596 & 18.6062 & 29.6763 & 30.2885 & 30.6550 \\
\hline MHR4 & 13.9395 & 18.3181 & 24.6705 & 30.3678 & 30.5640 & 33.9737 \\
\hline MHR \pm & 3.1738 & 5.1859 & 5.5979 & 7.0148 & 7.7790 & 9.1996 \\
\hline MHR4士 & 8.1940 & 9.0023 & 10.5224 & 12.2961 & 12.5958 & 14.3421 \\
\hline PP23 & 3.1836 & 5.2373 & 5.6681 & 7.1282 & 8.2192 & 9.5185 \\
\hline $\mathrm{ZZ}$ & 3.1649 & 5.1612 & 5.5674 & 6.9544 & 7.8132 & 9.1460 \\
\hline HSDT_32 & 3.3692 & 5.6590 & 7.2343 & 8.6884 & 9.1652 & 11.4630 \\
\hline HSDT & 4.9605 & 7.6850 & 8.5441 & 10.4062 & 10.9805 & 13.0905 \\
\hline FSDT & 12.1820 & 19.4765 & 23.1196 & 27.6664 & 28.0848 & 34.2690 \\
\hline 7 & X3, ZZ & $A-X X, \overline{Z Z}$ & error $<1$ & אחק גרד & D & ZA_X2, \\
\hline
\end{tabular}



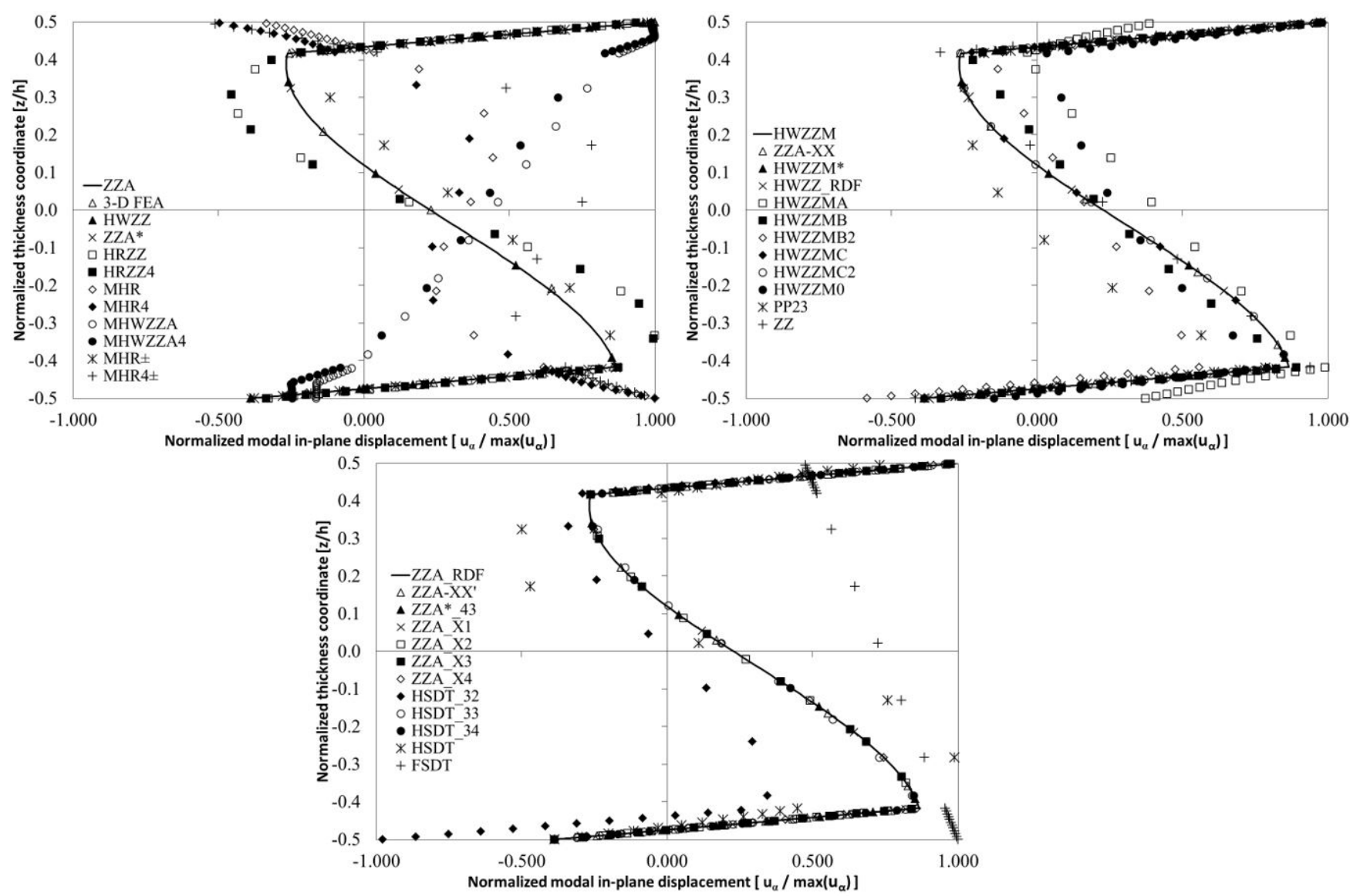

Figure 2 Case d: Normalized modal in-plane displacement (mode 4, bending).

First, it is noted from Table 8 that there are no pumping modes among the first considered, due to the combination of properties of faces and core considered, although there are not significant changes compared to the previous case. Consequently, even most of lower-order models can accurately predict eigen-frequencies and eigenmodes, the only exception being represented by MHWZZA4, MHR, MHR4, MHR4 \pm , HSDT_32, HSDT and FSDT theories.

Higher-order theories without zig-zag functions, i.e. HSDT_34, ZZA* 43, ZZA*, ZZA_X1 to _X4, HWZZM and HWZZM*, appear convenient for this case, as they combine accuracy and low costs, but also HWZZ appears accurate and efficient. All the other theories work very well, a sign that this case presents a return to characteristics similar to those of the first two cases examined where the effects of transverse normal deformation are not of primary importance.

As examples, the modal distribution of $u_{\alpha}$ and $\sigma_{\alpha \varsigma}$ across the thickness are reported in Figures 2 and 3, which confirm the previous considerations. The following additional cases are examined in search of behaviors that may highlight further peculiarities of theories. All the final considerations of the previous case $d$ still apply as regards the theoretical aspects of modeling concerning the choice of zig-zag functions, their omission, the choice of the representation and how it can be varied without results change, as well as the role to be assigned to displacement field coefficients and the fact that certain lower-order theories can be sufficiently accurate.

Case e. This case proposes a denser and more rigid core than case $c$ and two laminated faces made of epoxy glass and epoxy rayon layers (see, Table 4). Therefore, this case has strong different material properties which increase layerwise effects rising due to different stiffness ratios and material properties of faces and core. In this case pumping modes start to take place from the eighth up to the tenth mode, as it results from Table 9 which shows the free vibration frequencies predicted by the various theories. These results show that almost all lower-order theories considered in this paper are unsuitable for accurately predicting the behavior in this case with strong layerwise effects, which therefore turns out to be decidedly challenging for structural models. In fact, theories HWZZMA, HWZZMO, MHWZZA, MHWZZA4, MHR4 \pm , MHR, MHR4, HSDT32. HSDT and FSDT appear totally inadequate being unable to predict even the first fundamental frequency with some accuracy, while HWZZMB, HWZZMB2, HRZZ, HRZZ4, MHR \pm are able to accurately capture modes only up to the seventh one, that is excluding all the pumping modes. 

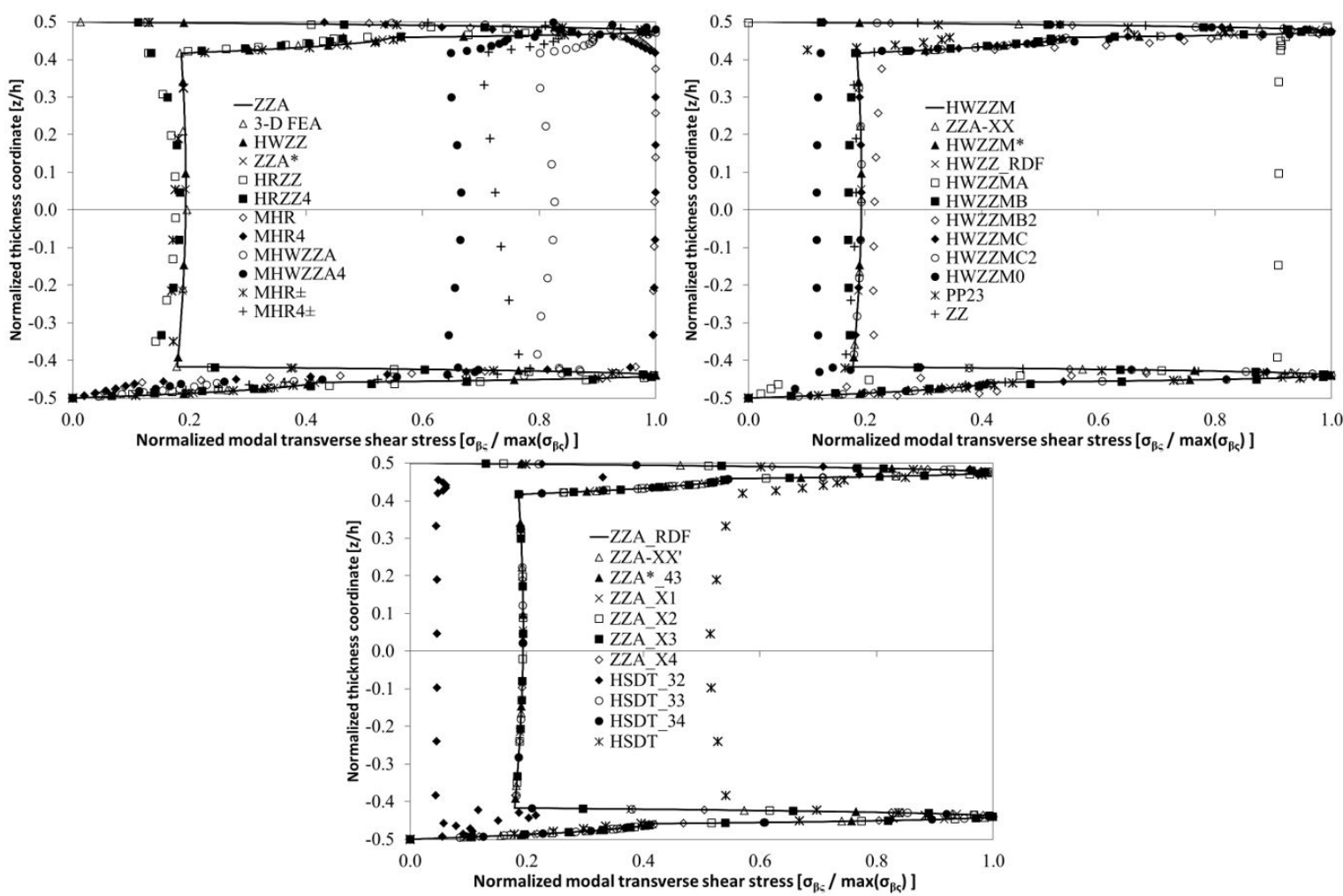

Figure 3 Case d: Normalized modal transverse shear stress (mode 4, bending).

Table 9 Normalized frequencies, case e.

\begin{tabular}{|c|c|c|c|c|c|c|c|c|c|c|}
\hline THEORY & $\begin{array}{c}\text { Mode } 1 \\
(1,1)\end{array}$ & $\begin{array}{c}\text { Mode } 2 \\
(1,2)\end{array}$ & $\begin{array}{c}\text { Mode } 3 \\
(2,1)\end{array}$ & $\begin{array}{c}\text { Mode } 4 \\
(1,3)\end{array}$ & $\begin{array}{c}\text { Mode } 5 \\
(2,2)\end{array}$ & $\begin{array}{c}\text { Mode } 6 \\
(2,3)\end{array}$ & $\begin{array}{c}\text { Mode } 7 \\
(1,4)\end{array}$ & $\begin{array}{c}\text { Mode } 8 \\
(1,2)\end{array}$ & $\begin{array}{c}\text { Mode } 9 \\
(1,3)\end{array}$ & $\begin{array}{c}\text { Mode } 10 \\
(1,1)\end{array}$ \\
\hline $\begin{array}{l}\text { 3D FEA } \\
\text { (2004) }\end{array}$ & 1.8250 & 2.9115 & 3.8103 & 4.3378 & 4.5581 & 5.7563 & 6.1051 & 6.4866 & 6.6342 & 6.7820 \\
\hline $\begin{array}{l}\text { Theories with } \\
\text { identical results }\end{array}$ & 1.8302 & 2.9306 & 3.8184 & 4.3758 & 4.5813 & 5.7983 & 6.1442 & 6.5052 & 6.6158 & 6.8955 \\
\hline HWZZMA & 5.6059 & 6.0176 & 7.4697 & 8.5589 & 16.2177 & 17.6828 & 18.2564 & 19.0662 & 36.9624 & 49.9279 \\
\hline HWZZMB & 1.8359 & 2.9415 & 3.8693 & 4.3991 & 4.6093 & 5.8330 & 6.1905 & 15.8263 & 27.0305 & 38.2535 \\
\hline HWZZMB2 & 1.8515 & 3.3472 & 4.0131 & 4.4161 & 4.6227 & 5.8334 & 6.1915 & 16.1119 & 29.0452 & 44.5313 \\
\hline HWZZMO & 2.5444 & 5.4380 & 6.2492 & 8.9994 & 10.6722 & 14.0410 & 27.9576 & 15.8951 & 27.3268 & 39.4091 \\
\hline HRZZ & 1.8116 & 2.8521 & 3.6589 & 4.1144 & 4.2849 & 5.1317 & 5.3547 & 13.3111 & 15.6067 & 22.8002 \\
\hline HRZZ4 & 1.8160 & 2.8702 & 3.6892 & 4.1721 & 4.3456 & 5.2736 & 5.5271 & 15.6428 & 16.6538 & 22.9738 \\
\hline MHWZZA & 0.6942 & 2.3977 & 2.6214 & 3.0188 & 3.9030 & 4.5651 & 6.1380 & 7.7168 & 10.9624 & 13.5256 \\
\hline MHWZZA4 & 0.6980 & 2.0240 & 2.4328 & 3.2701 & 3.4373 & 3.6584 & 3.8426 & 6.2970 & 21.3296 & 28.1332 \\
\hline MHR & 9.5006 & 12.2412 & 15.1366 & 17.2619 & 17.6349 & 17.9766 & 18.3084 & 18.8505 & 37.6813 & 52.1934 \\
\hline MHR4 & 9.5609 & 13.3372 & 18.6770 & 18.9136 & 20.7667 & 24.6293 & 25.1072 & 15.6264 & 25.7609 & 34.6050 \\
\hline MHR \pm & 1.8389 & 2.9673 & 3.8584 & 4.4778 & 4.6501 & 5.9317 & 6.3553 & 16.9912 & 31.6639 & 45.3776 \\
\hline MHR4 \pm & 5.0499 & 5.9727 & 6.7604 & 7.1795 & 7.2376 & 7.8740 & 7.9708 & 31.6309 & 36.3794 & 41.6820 \\
\hline PP23 & 1.8537 & 3.0414 & 4.0033 & 4.8769 & 4.8892 & 6.4580 & 7.3601 & 10.6373 & 10.8652 & 11.9880 \\
\hline $\mathrm{ZZ}$ & 1.8612 & 2.9504 & 4.0880 & 4.3963 & 4.8128 & 5.9917 & 6.1812 & 7.6125 & 7.6180 & 7.8116 \\
\hline HSDT_32 & 2.0354 & 3.1806 & 4.8855 & 5.2842 & 6.0359 & 7.1412 & 7.3720 & 7.5825 & 8.7272 & 12.6639 \\
\hline HSDT_33 & 1.8301 & 2.9303 & 3.8175 & 4.3750 & 4.5800 & 5.7964 & 6.1428 & 6.7578 & 6.8938 & 7.1157 \\
\hline HSDT & 3.3835 & 5.1523 & 6.0926 & 7.2858 & 7.3239 & 9.0389 & 9.7357 & - & - & - \\
\hline FSDT & 8.7966 & 13.4532 & 17.2541 & 19.7304 & 20.0760 & 24.7239 & 26.4143 & - & - & - \\
\hline
\end{tabular}

- ZZA, ZZA*, HWZZ, HWZZM, HWZZM*, HWZZMC, HWZZMC2, HSDT_34, ZZA*_43, ZZA_RDF, HWZZ_RDF, ZZA_X1, ZZA_X2, ZZA_X3, ZZA_X4, ZZA-XX, ZZA-XX' (error $<1 \%$ ); Colored columns: pumping modes. 
This highlights once again a diversification of the type of performance achievable by the various theories depending on the case considered. In this case, theories PP23 (whose displacement field is parabolic-cubic), and ZZ (which does not consider the redefinition of coefficients) appear much less accurate than in the previous cases, a sign that only theories that impose the fulfillment of full set off physical constraint $(6,8-11)$ and whose terms are redefined, possess the ability to adapt to changing conditions across the thickness caused by layerwise effects.

Furthermore, it is seen that as the frequency of modes increases, lower-order theories make ever greater errors, so the only fundamental frequency is not sufficient to test the accuracy of structural models, as well known but not always taken into due consideration in the literature.

For this case the variation of $\sigma_{\varsigma \varsigma}$ across the thickness for mode 10 is given as an example in Figure 4, for which the following considerations that can also be extended to its other two out-of-plane stress counterparts apply. The fundamental aspect to note is that the correct variation of modal stresses across the thickness is captured in this case only by higher order theories.
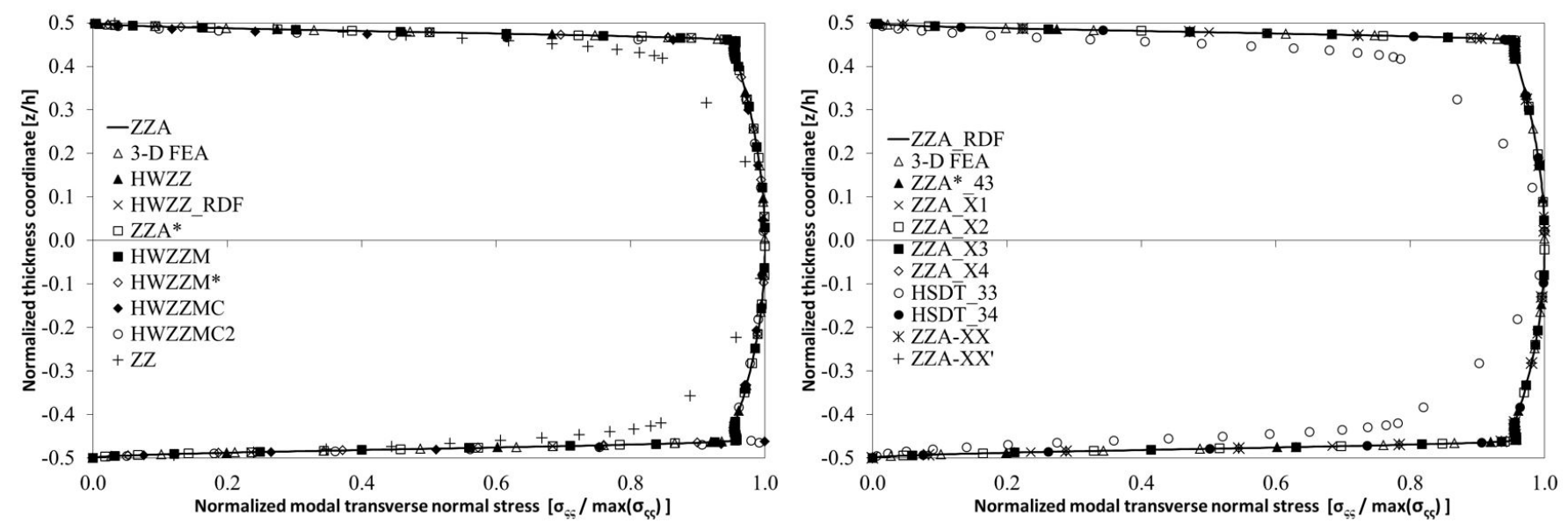

Figure 4 Case e: Normalized modal transverse normal stress (mode 10, pumping).

The same holds for displacements, which in non-mixed theories directly affect the stresses, but also the mixed formulations of this paper, wherein stresses are assumed apart with the intention of obtaining greater accuracy, appear however inadequate. Anyway it could be noticed that HSDT_33, HWZZMC, C2 and ZZ, even if not included among the higher-order theories, in this case prove to be adequate.

Beyond this, the numerical efficiency of high-order theories is confirmed (see Table 3), along with that zig-zag functions can be arbitrarily chosen, or even that they can be omitted, as well as the form of representation can be assumed arbitrarily, without the results being affected, so confirming the results of previous cases.

Case $\mathbf{f}$. This case derives from the previous one considering a more rigid and denser core (see, Table 4) selected between available foamy materials of industrial interest, while the faces are the same made of layers of epoxy glass and epoxy rayon. Due to the variation of the core characteristics remarked above, a different behavior than for the previous case is shown by the results of Table 10 regarding free vibration frequencies, because similarly to case $d$ but differently to previous case e the existence of pumping modes does not occur among the first 10 modes.

As a consequence, the theories exhibit a dissimilar behavior compared to that shown for the previous case e because the dispersion of the results is lower, despite apparently there should be the same layerwise effects as the characteristics of the core assumed are still similar to those of case e, while in reality they are minor since the existence of pumping modes is precluded. As a consequence, for this case, which further demonstrates that even mild variations of material properties cause strongly different behaviors in sandwich structures, many theories appear adequate.

In fact, the first frequencies are captured with sufficient precision by most theories, the exceptions being represented by HWZZMA, HWZZM0, MHWZZA, MHWZZA4, MHR, MHR4, MHR4 £, HSDT, FSDT, HSDT_32. As many of the considerations of case e still holds and discrepancies among theories are more limited than in the previous case, for the present case are not reported figures.

As far as calculation costs (Table 3) and theoretical aspects of modeling are concerned, what has already been observed in the previous case about the choice of zig-zag functions and of the representation form across the thickness is confirmed. 
Table 10 Normalized frequencies, case $f$.

\begin{tabular}{|c|c|c|c|c|c|c|c|c|c|c|}
\hline THEORY & $\begin{array}{c}\text { Mode } 1 \\
(1,1)\end{array}$ & $\begin{array}{c}\text { Mode } 2 \\
(1,2)\end{array}$ & $\begin{array}{c}\text { Mode } 3 \\
(2,1)\end{array}$ & $\begin{array}{c}\text { Mode } 4 \\
(1,3)\end{array}$ & $\begin{array}{c}\text { Mode } 5 \\
(2,2)\end{array}$ & $\begin{array}{c}\text { Mode } 6 \\
(2,3)\end{array}$ & $\begin{array}{c}\text { Mode } 7 \\
(1,4)\end{array}$ & $\begin{array}{c}\text { Mode } 8 \\
(3,1)\end{array}$ & $\begin{array}{c}\text { Mode } 9 \\
(3,2)\end{array}$ & $\begin{array}{c}\text { Mode } 10 \\
(2.4)\end{array}$ \\
\hline 3D FEA'Icardi and Atzori (2004) & 2.0795 & 3.3263 & 4.1141 & 4.9108 & 4.9759 & 6.3078 & 6.8086 & 7.4053 & 8.0236 & 8.0545 \\
\hline $\begin{array}{c}\text { Theories with } \\
\text { identical results }\end{array}$ & 2.0796 & 3.3265 & 4.1004 & 4.9036 & 4.9648 & 6.2918 & 6.7699 & 7.3228 & 7.9472 & 8.0112 \\
\hline HWZZMA & 5.5699 & 6.0388 & 7.2736 & 8.6068 & 16.2809 & 16.6222 & 17.1914 & 17.4915 & 30.6469 & 28.1735 \\
\hline HWZZMB & 2.0847 & 3.3368 & 4.1449 & 4.9246 & 4.9909 & 6.3213 & 6.8051 & 7.4984 & 8.0336 & 8.0493 \\
\hline HWZZMB2 & 2.1027 & 3.4048 & 4.3459 & 4.9246 & 5.3958 & 6.3213 & 6.8051 & 7.8723 & 8.0487 & 8.0962 \\
\hline HWZZMO & 2.6805 & 5.5223 & 6.3050 & 8.9939 & 10.6038 & 12.3649 & 13.8818 & 14.4314 & 17.7913 & 20.3025 \\
\hline HRZZ & 2.0555 & 3.2287 & 3.9386 & 4.6061 & 4.6658 & 5.6683 & 6.0051 & 6.2595 & 6.4412 & 6.6360 \\
\hline HRZZ4 & 2.0616 & 3.2539 & 3.9753 & 4.6830 & 4.7377 & 5.8248 & 6.2043 & 6.4185 & 6.6973 & 6.9919 \\
\hline MHWZZA & 0.6897 & 2.5190 & 2.8355 & 3.2094 & 4.0947 & 4.7940 & 5.2636 & 6.3369 & 10.2714 & 11.2195 \\
\hline MHWZZA4 & 0.6916 & 2.1904 & 2.5936 & 3.4786 & 3.6347 & 3.8856 & 4.0466 & 5.6961 & 8.6553 & 8.7156 \\
\hline MHR & 9.2783 & 11.9900 & 14.8886 & 17.0729 & 17.2357 & 17.6067 & 18.5309 & 19.4935 & 21.3978 & 21.7227 \\
\hline MHR4 & 9.3355 & 13.0453 & 18.2477 & 18.5238 & 20.3075 & 24.1139 & 24.6192 & 26.5227 & 28.1148 & 29.0030 \\
\hline MHR \pm & 2.0873 & 3.3592 & 4.1351 & 4.9964 & 5.0251 & 6.4125 & 6.9726 & 7.4450 & 8.0906 & 8.2465 \\
\hline MHR4 \pm & 5.1728 & 6.5043 & 7.5808 & 7.6859 & 7.8535 & 8.8055 & 9.2334 & 8.2442 & 8.9815 & 10.1505 \\
\hline PP23 & 2.1040 & 3.4462 & 4.2652 & 5.2636 & 5.4763 & 6.9835 & 8.0887 & 8.1308 & 8.9025 & 9.4691 \\
\hline $\mathrm{ZZ}$ & 2.1040 & 3.3412 & 4.3350 & 4.9154 & 5.1633 & 6.4531 & 6.7886 & 8.0586 & 8.1505 & 8.6417 \\
\hline HSDT_32 & 2.2404 & 3.5104 & 5.2991 & 5.4004 & 6.2323 & 7.5868 & 7.6496 & 9.6758 & 10.9591 & 11.5818 \\
\hline HSDT & 3.4532 & 5.2974 & 6.1860 & 7.4331 & 7.5539 & 9.2594 & 9.6431 & 10.0533 & 10.5555 & 11.4844 \\
\hline FSDT & 8.5977 & 13.1629 & 16.8641 & 19.3114 & 19.6340 & 24.1875 & 25.2568 & 25.8551 & 27.2188 & 29.6662 \\
\hline
\end{tabular}

- ZZA, ZZA*, HWZZ, HWZZM, HWZZM*, HWZZMC, HWZZMC2, HSDT_33, HSDT_34, ZZA*_43, ZZA_RDF, HWZZ_RDF, ZZA_X1, ZZA_X2, ZZA_X3, ZZA_X4, ZZA-XX, ZZA-XX' (error $<1 \%)$.

Case $g$. This case has the same laminated faces of cases e and $f$, made of epoxy-glass and epoxy-rayon layers, but currently the core is made up of two different industrial foams, one constituting the $3 / 4$ of the thickness which is made of Rohacell 31 and the other for the remaining quarter which it is made by the same manufacturer but which is less rigid but more dense (properties given in Table 4). In this way the sandwich structure takes on characteristics that are even more strongly asymmetrical than those of case e due to the faces, which make the present case very challenging from the standpoint of modeling.

So, differences between the predictions of the various theories are more marked, as shown by the results for the free vibration frequencies presented in Table 11. $u_{\beta}$ and $u_{\varsigma}$ modal displacements of the first mode, are reported in Figures 5 and 6, respectively; it should be noticed that nevertheless figures concern a bending mode, strong discrepancies caused by the noticeable layerwise effects are noted among the results by the various theories.

In Figure 7 it is also reported the through-thickness variation of $\sigma_{\varsigma \varsigma}$ for pumping mode 9, which provides a direct measure of the ability of theories to describe the transverse normal deformation. Because of considerable discrepancies between theories, only higher order theories and HWZZMC, HWZZMC2, HSDT_33 (that commit errors of less than $3 \%$ ) and ZZ (that is still sufficiently accurate, but whose errors are over $10 \%$ ) are reported in Figure 7 . Because of coefficients of ZZ are defined once and for all layers, once again the beneficial role played by the redefinition of coefficients is highlighted. The ZZ model of 2001 despite having fixed coefficients appears superior to many mixed models like MHR, MHR4, MHWZZA, HWZZMA, HWZZMO, HSDT_32, because although it requires a post-processing it appears cost-effective and more easy to implement. So ZZ represents a valid alternative for not particularly challenging cases that do not require the use of adaptive models.

Instead of other cases, theories HWZZMA, HWZZMB, HWZZMB2, HWZZMO, MHWZZA, MHWZZA4, MHR, MHR4, MHR \pm , MHR4 \pm , HSDT_32 now provide highly inaccurate results, sign that when layerwise effects are strongly enhanced these theories are unsuited. Since PP23, ZZ, HSDT_33 appear less accurate than adaptive theories with redefined coefficients at the highest frequency considered, it is proven that only by cubic / quartic theories whose coefficients are redefined through the thickness are appropriate for higher-order modes. 
Table 11 Normalized frequencies, case g.

\begin{tabular}{|c|c|c|c|c|c|c|c|c|c|c|}
\hline THEORY & $\begin{array}{c}\text { Mode } 1 \\
(1,1)\end{array}$ & $\begin{array}{c}\text { Mode } 2 \\
(1,2)\end{array}$ & $\begin{array}{c}\text { Mode } 3 \\
(2,1)\end{array}$ & $\begin{array}{c}\text { Mode } 4 \\
(1,3)\end{array}$ & $\begin{array}{c}\text { Mode } 5 \\
(2,2)\end{array}$ & $\begin{array}{c}\text { Mode } 6 \\
(2,3)\end{array}$ & $\begin{array}{c}\text { Mode } 7 \\
(1,2)\end{array}$ & $\begin{array}{c}\text { Mode } 8 \\
(1,4)\end{array}$ & $\begin{array}{c}\text { Mode } 9 \\
(1,1)\end{array}$ & $\begin{array}{c}\text { Mode } 10 \\
(1,3)\end{array}$ \\
\hline 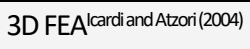 & 1.8088 & 2.8905 & 3.8226 & 4.3159 & 4.5659 & 5.7612 & 5.8607 & 6.0359 & 6.0584 & 6.1857 \\
\hline $\begin{array}{l}\text { Theories with } \\
\text { identical results }\end{array}$ & 1.8078 & 2.8887 & 3.7844 & 4.3068 & 4.5319 & 5.7260 & 5.8201 & 6.0452 & 6.0635 & 6.1789 \\
\hline HWZZMA & 5.6747 & 7.4231 & 11.1292 & 14.2994 & 16.4451 & 17.3334 & 17.3941 & 19.7369 & 21.3192 & 22.6575 \\
\hline HWZZMB & 2.8387 & 5.7485 & 7.5786 & 9.8365 & 9.9593 & 13.7832 & 15.7482 & 16.2849 & 19.0510 & 29.2717 \\
\hline HWZZMB2 & 2.6087 & 5.5092 & 6.6212 & 9.3733 & 9.8365 & 10.2068 & 13.7832 & 15.8463 & 19.0510 & 29.2851 \\
\hline HWZZMO & 3.0359 & 6.5841 & 7.6661 & 10.7550 & 12.5084 & 15.7974 & 16.2958 & 16.3219 & 20.4687 & 29.5538 \\
\hline HRZZ & 1.8069 & 2.8837 & 3.7376 & 4.2738 & 4.4553 & 5.5480 & 15.8071 & 5.8823 & 28.4136 & 28.7126 \\
\hline HRZZ4 & 1.8067 & 2.8826 & 3.7339 & 4.2671 & 4.4484 & 5.5302 & 15.6286 & 5.8443 & 27.3772 & 27.9550 \\
\hline MHWZZA & 0.7246 & 0.9179 & 3.3407 & 3.3479 & 3.5688 & 4.3557 & 6.1238 & 4.0545 & 7.9043 & 16.7350 \\
\hline MHWZZA4 & 0.6978 & 0.8651 & 3.5557 & 6.3966 & 6.8917 & 9.7175 & 6.2385 & 10.1841 & 6.8404 & 23.3498 \\
\hline MHR & 10.9702 & 12.9657 & 15.3170 & 17.2175 & 19.5764 & 19.5866 & 12.4103 & 20.1339 & 12.9515 & 25.9401 \\
\hline MHR4 & 11.1870 & 14.7950 & 22.6656 & 22.9203 & 26.2358 & 33.1135 & 15.4831 & 34.9702 & 27.0792 & 28.0628 \\
\hline MHR \pm & 2.1762 & 3.5080 & 4.3617 & 5.2810 & 5.3128 & 6.8076 & 20.4583 & 7.3859 & 27.0941 & 39.0589 \\
\hline MHR4 \pm & 2.4204 & 4.0933 & 4.8042 & 5.9706 & 6.1081 & 7.6269 & 25.1892 & 8.3280 & 29.2856 & 48.0530 \\
\hline PP23 & 1.8240 & 2.9766 & 3.9065 & 4.6939 & 4.7518 & 6.2354 & 7.0409 & 7.2868 & 7.7506 & 8.9504 \\
\hline $\mathrm{ZZ}$ & 1.8390 & 2.9088 & 4.0557 & 4.3283 & 4.7655 & 5.9222 & 6.0851 & 6.5598 & 6.5919 & 7.9180 \\
\hline HSDT_32 & 2.8859 & 5.3262 & 6.8437 & 8.8258 & 9.9568 & 34.1067 & 41.9080 & 42.6294 & 50.1473 & 53.2910 \\
\hline HSDT_33 & 1.8076 & 2.8884 & 3.7832 & 4.3069 & 4.5306 & 5.7250 & 5.8278 & 6.0465 & 6.3980 & 6.4500 \\
\hline HSDT & 3.3705 & 5.1307 & 6.0685 & 7.2555 & 7.2900 & 8.9979 & - & 9.6855 & - & - \\
\hline FSDT & 8.7728 & 13.4157 & 17.2080 & 19.6752 & 20.0217 & 24.6560 & - & 26.3410 & - & - \\
\hline
\end{tabular}

- ZZA, ZZA*, HWZZ, HWZZM, HWZZM*, HWZZMC, HWZZMC2, HSDT_34, ZZA*_43, ZZA_RDF, HWZZ_RDF, ZZA_X1, ZZA_X2, ZZA_X3, ZZA_X4, ZZA-XX, ZZA-XX' (error < 1\%); Dashed lines: pumping modes.
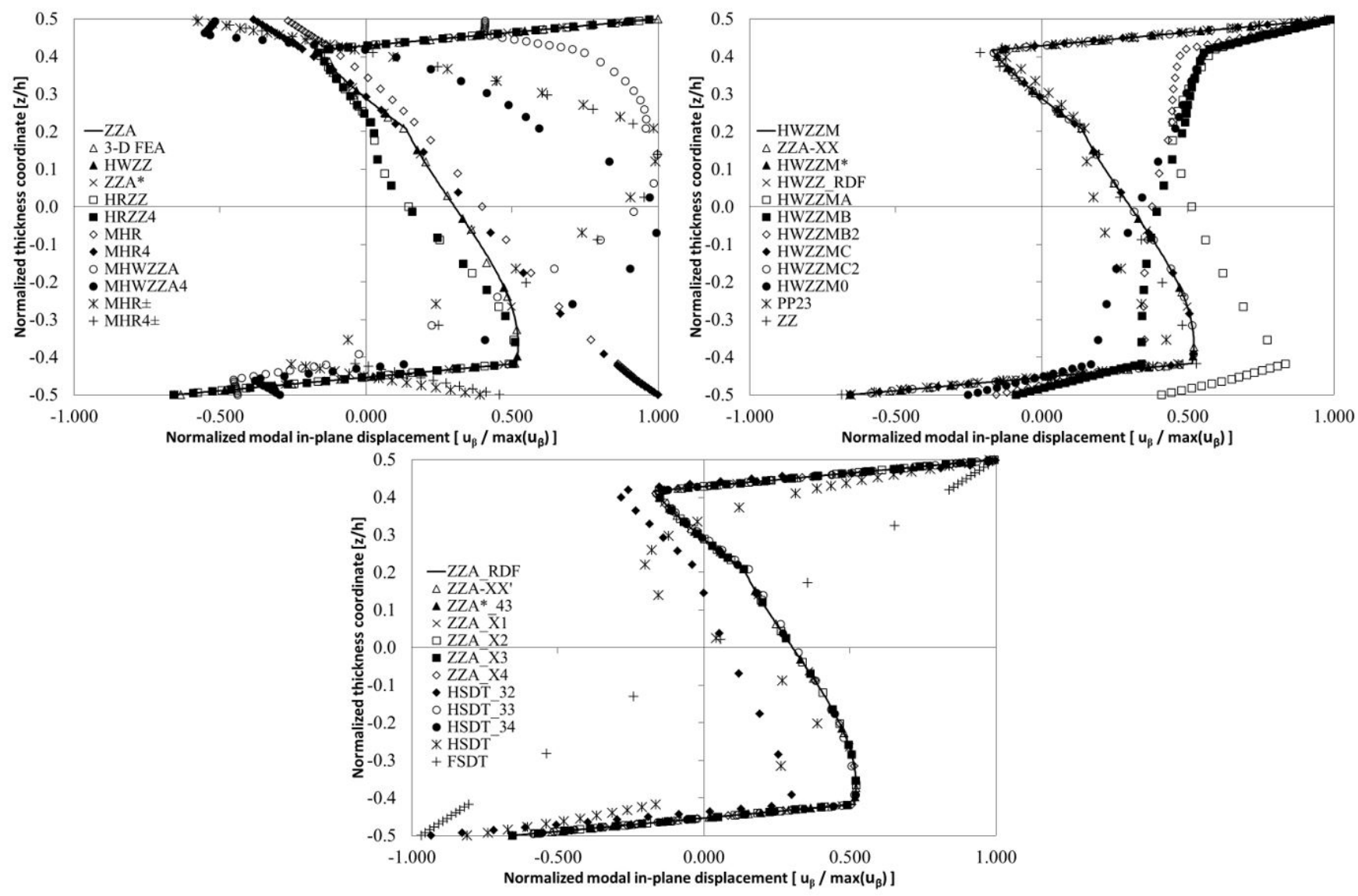

Figure 5 Case g: Normalized modal in-plane displacement (mode 1, bending). 

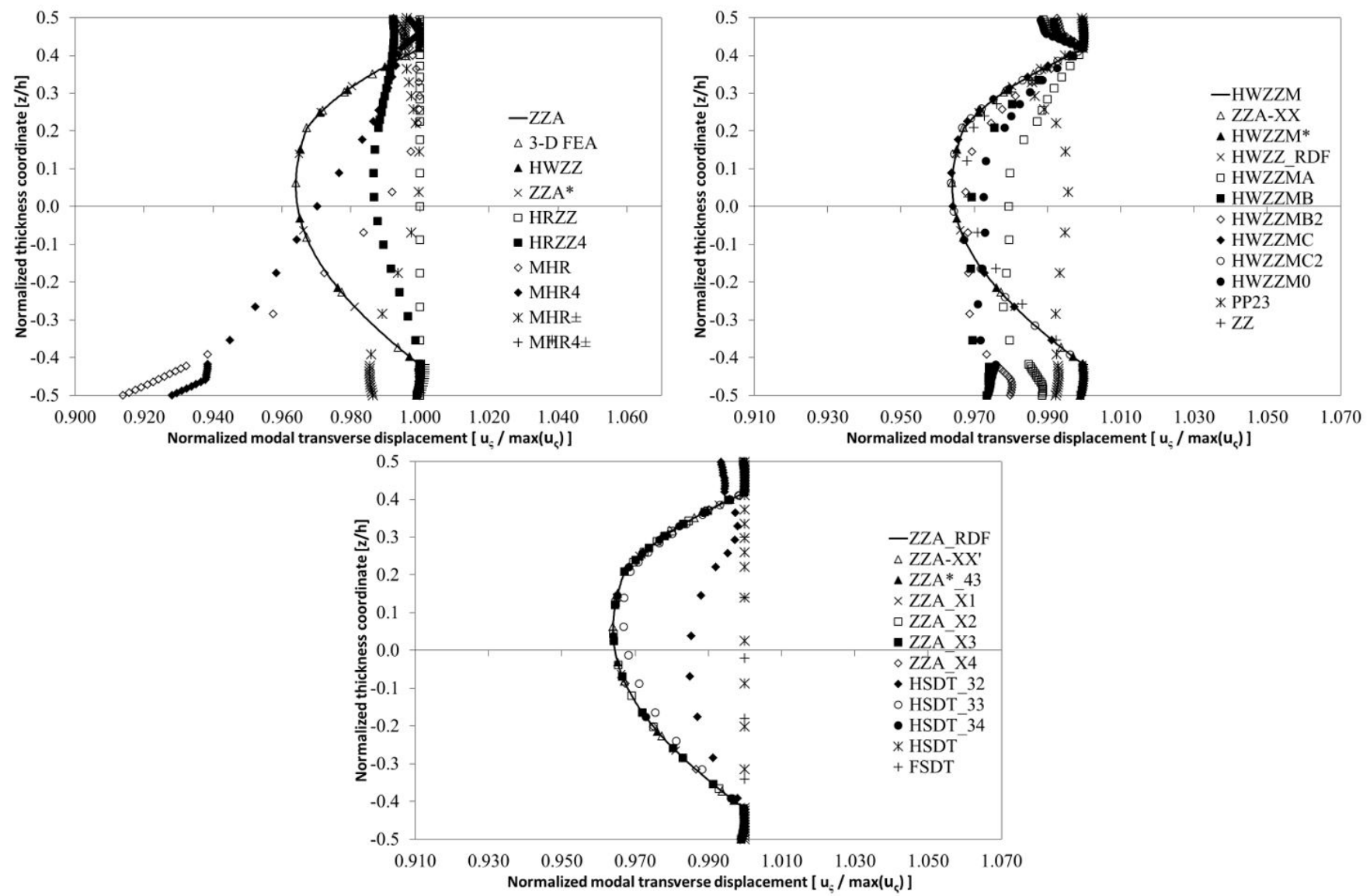

Figure 6 Case g: Normalized modal transverse displacement (mode 1, bending).
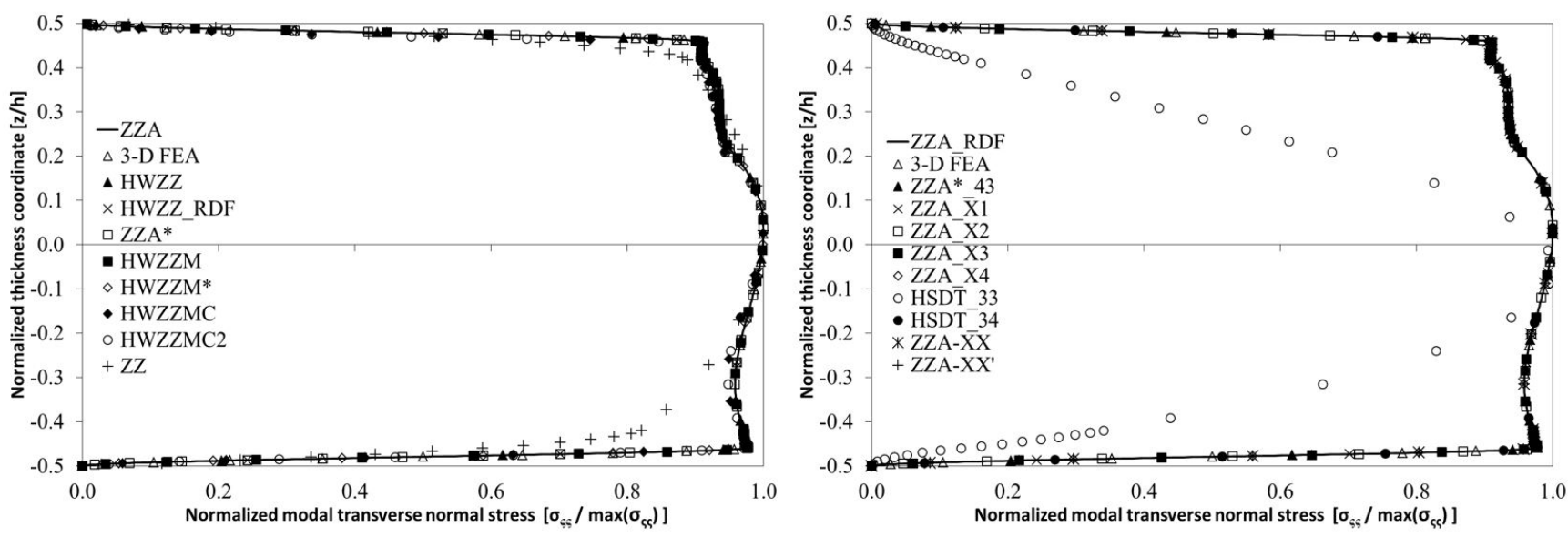

Figure 7 Case g: Normalized modal transverse normal stress (mode 9, pumping).

Because of all this, it turns out that mixed theories such as HRZZ, HRZZ4, MHWZZA, MHWZZA4 (first ones with characteristics similar to those of theories proposed in the literature) has little practical significance since they are neither particularly advantageous from the standpoint point of computational costs savings, nor adequate to study vibrations due to a displacement field not well represented, although the stress field can be quite well represented.

The results also show that theories HWZZMC and HWZZMC2 can accurately calculate natural frequencies, while they are less accurate in calculating modal displacements and stresses.

The always accurate results of the adaptive theories of higher order ZZA, ZZA *, HWZZM, HWZZM *, HWZZ, HSDT_34, ZZA * _43, ZZA-XX, ZZA-XX ', ZZA_RDF, HWZZ_RDF and ZZA_X1, to _X4, demonstrate what claimed by the theoretical viewpoint at the beginning of section 4 dedicated to the numerical results, as well as in the introductory section, i.e. about the possibility of arbitrarily choosing the zig-zag functions and the form of the representation. 
Whoever studies problems involving sandwiches in the literature in an analytical way usually uses symmetric laminations, because the theories developed for these structures usually assume the symmetry. Then the finite element method the used for studying asymmetric sandwiches which may exist due to project choices or due to the occurrence of damage, for example due to impact. But the results obtained for this case show that the present higherorder models are as accurate as 3-D FEA,although they have the same number of variables as classical theories like FSDT and HSDT, so they can be used as an alternative to more expensive models in terms of cost and preparation without loss of accuracy even for asymmetric laminations.

\section{CONCLUDING REMARKS}

In this paper, free vibrations of flexible soft-core sandwich structures assumed as multilayer structures having strong variations of properties of constituent laminae are studied, with the intended aim to discriminate which theoretical assumptions, meaning which different representation of variables across the thickness and which different zig-zag functions, can be more appropriate to efficiently and accurately capture pumping modes dominated by the transverse normal deformation.

Various zig-zag theories by the authors, having a fixed number of variables irrespective of the number of constituent layers, a different representation across the thickness and different zig-zag functions, have been considered in order to confirm the findings of previous articles, at the time demonstrated for static applications and to find other new ones characteristic of physically based theories whose coefficients are redefined across the thickness by enforcing the physical constraints imposed by the elasticity theory.

The intended aim is to numerically prove that, whenever the full set of physical constraints is satisfied, the choice of zig-zag functions is immaterial, that these functions can even be omitted if coefficients, whose number is related to the number of constraints to enforce, are re-determined at interfaces, and moreover that the order of representation of in-plane and transverse displacement components can be freely assumed, as well as that the role assigned to single coefficients because results remains unchanged. In addition, it is proven that there is no variation in the accuracy of results exchanging the order of representation of in-plane and transverse displacement components, while if physical constraints are only partially satisfied the accuracy lowers and becomes dependent on the assumptions made, so the choice of zig-zag and representation functions can no longer be arbitrary.

Confirming the findings in the literature, numerical applications show that pumping modes, for which the transverse deformation becomes as important as transverse shear and in-plane ones, may or may not appear among the first mode, as the behaviour is strongly dependent on the material properties of core and faces of the individual cases.

Certain lower-order theories with only a partial fulfillment of constraints can still be sufficiently accurate in predicting pumping modes, as well as modal through-thickness displacement and stress fields, but in most cases the only rule that seems satisfied and therefore assumes a general character is that only higher-order theories with a full fulfilment of physical constraints can always accurately and efficiently capture the effects played by the transverse normal deformation and so to adequately represent the dynamic behavior.

Considering that their computational cost is still comparable with that of lower-order counterparties which are not always accurate, they turn out to be the most suited ones for analysis. However, only those with the highest rank, i.e. with no simplifying assumptions, are such. Indeed, discrepancies appear among theories with a higher-order representation and reference results, when formulated by introducing simplifying assumptions, without a general rule being drawn from theories that fail because they are not always the same in the various cases examined.

It turns out from numerical results that mixed theories of this paper, some of which have characteristics similar to those of theories proposed in the literature, have little practical significance since they are neither advantageous from the standpoint point of computational costs saving, nor accurate as their displacement field is not well represented.

The results also show that the present, most accurate higher-order models reach the same accuracy degree of 3-D FEA with a much lower number of unknowns and ultimately with a much lower cost, both because an analytical approach is used and the d.o.f. number is the same as classical theories like FSDT and HSDT. So the theories of this paper can be used as an alternative to more expensive models in terms of cost and preparation without any loss of accuracy.

Finally, the results confirm what claimed above about the arbitrariness of zig-zag and representation functions if the coefficients are redefined in order to satisfy all the physical constraints required by the elasticity theory. It is also shown that the redefinition of coefficients allows the theories of any order to significantly increase their accuracy and, in addition that exchanging order and form of representation results do not change. 
Author's Contributions: Conceptualization, U Icardi and A Urraci; Methodology, U Icardi and A Urraci; Investigation, U Icardi and A Urraci; Writing - original draft, U Icardi and A Urraci; Writing - review \& editing, U Icardi and A Urraci; Supervision, U Icardi and A Urraci.

Editor: Rogério José Marczak.

\section{References}

Altenbach, H. (1998). Theories for laminated and sandwich plates. A review, Mechanics of Composite Materials 34(3):243-252.

Barouni, A.K., Saravanos, D.A. (2016). A layerwise semi-analytical method for modeling guided wave propagation in laminated and sandwich composite strips with induced surface excitation, Aerospace Science and Technology 51:118-141.

Boscolo, M., Banerjee, J.R. (2014). Layer-wise dynamic stiffness solution for free vibration analysis of laminated composite plates, Journal of Sound and Vibration 333(1):200-227.

Burlayenko, V.N., Altenbach, H., Sadowski, T. (2015). An evaluation of displacement-based finite element models used for free vibration analysis of homogeneous and composite plates, Journal of Sound and Vibration 358:152-175.

Carrera, E. (1999). A study of transverse normal stress effects on vibration of multilayered plates and shells, Journal of Sound and Vibration 225:803-829.

Carrera, E. (2001). Developments, ideas, and evaluations based upon Reissner's mixed variational theorem in the modeling of multilayered plates and shells, Applied Mechanics Reviews 54(4):301-329.

Carrera, E. (2003). Historical review of zig-zag theories for multilayered plates and shells, Applied Mechanics Reviews 56(3):287-308

Carrera, E. (2004). On the use of the Murakami's zig-zag function in the modeling of layered plates and shells, Computers \& Structures 82(7-8):541-554.

Carrera, E., Ciuffreda, A. (2005). Bending of composites and sandwich plates subjected to localized lateral loadings: a comparison of various theories, Composite Structures 68:185-202.

Catapano, A., Giunta, G. Belouettar, S. Carrera, E. (2011). Static analysis of laminated beams via a unified formulation, Composite Structures 94(1):75-83.

Cho, K.N., Bert, C.W., Striz, A.G. (1991). Free vibrations of laminated rectangular analyzed by higher order individual-layer theory, Journal of Sound and Vibration 145(3):429-442.

de Miguel, A.G., Carrera, E., Pagani, A., Zappino, E. (2018) Accurate Evaluation of Interlaminar Stresses in Composite Laminates via Mixed One-Dimensional Formulation, AIAA Journal 56(11):4582-4594.

Demasi, L. (2004). Refined multilayered plate elements based on Murakami zig-zag functions, Composite Structures 70:308316 .

Di Sciuva, M. (1984). A refinement of the transverse shear deformation theory for multilayered orthotropic plates, L'Aerotecnica, Missili e Spazio 62:84-92.

Frostig, Y., Thomsen, O.T. (2004). High-order free vibration of sandwich panels with a flexible core, International Journal of Solids and Structures 41:1697-1724.

Frostig, Y., Thomsen, O.T. (2009a). On the free vibration of sandwich panels with a transversely flexible and temperaturedependent core material - Part I: Mathematical formulation, Composites Science and Technology 69:856-862.

Frostig, Y., Thomsen, O.T. (2009b). On the free vibration of sandwich panels with a transversely flexible and temperaturedependent core material- Part II: Numerical study, Composites Science and Technology 69:863-869.

Icardi, U. (2001). Higher-order zig-zag model for analysis of thick composite beams with inclusion of transverse normal stress and sublaminates approximations, Composites Part B: Engineering 32(4):343-354.

Icardi, U., Atzori, A. (2004). Simple, efficient mixed solid element for accurate analysis of local effects in laminated and sandwich composites, Advances in Engineering Software 35(12):843-859. 
Icardi, U., Sola, F. (2014). Development of an efficient zig-zag model with variable representation of displacements across the thickness, Journal of Engineering Mechanics 140(3):531-541.

Icardi, U., Urraci, A. (2018a). Free and forced vibration of laminated and sandwich plates by zig-zag theories differently accounting for transverse shear and normal deformability, Aerospace 5(4):108.

Icardi, U., Urraci, A. (2018b). Novel HW mixed zig-zag theory accounting for transverse normal deformability and lower-order counterparts assessed by old and new elastostatic benchmarks, Aerospace Science and Technology 80:541-571.

Jun, L., Xiang, H., Li Xiaobin, L. (2016). Free vibration analyses of axially loaded laminated composite beams using a unified higher-order shear deformation theory and dynamic stiffness method, Composite Structures 158:308-322.

Kapuria, S., Dumir, P.C., Jain, N.K. (2004). Assessment of zig-zag theory for static loading, buckling, free and forced response of composite and sandwich beams, Composite Structures 64(3-4):317-327.

Kapuria, S., Nath, J.K. (2013). On the accuracy of recent global-local theories for bending and vibration of laminated plates, Composite Structures 95:163-172.

Kazancı, Z. (2016). A review on the response of blast loaded laminated composite plate, Progress in Aerospace Sciences 81:4959.

Khandan, R., Noroozi, S., Sewell, P., Vinney, J. (2012). The development of laminated composite plate theories: a review, Journal of Materials Science 47(16):5901-5910.

Khdeir, A.A., Aldraihem, O.J. (2016). Free vibration of sandwich beams with soft core, Composite Structures 154:179-189.

Kim, J.S. (2007). Free vibration of laminated and sandwich plates using enhanced plate theories, Journal of Sound and Vibration 308(1-2):268-286.

Li, X.Y., Liu, D. (1997). Generalized laminate theories based on double superposition hypothesis, International Journal for Numerical Methods in Engineering 40:197-212.

Lin, T.R., Zhang K. (2018). An analytical study of the free and forced vibration response of a ribbed plate with free boundary conditions, Journal of Sound and Vibration 422:15-33.

Lopatin, A.V., Morozov, E.V. (2010). Symmetrical vibration modes of composite sandwich plates. Journal of Sandwich Structures and Materials 13(2):189-211.

Lur'e, S.A., Shumova, N.P. (1996). Kinematic models of refined theories concerning composite beams plates and shells, Mechanics of Composite Materials 32(5):422-430.

Malekzadeh, K., Khalili, M.R., Mittal, R.K. (2005). Local and Global Damped Vibrations of Plates with a Viscoelastic Soft Flexible Core: An Improved High-order Approach, Journal of Sandwich Structures \& Materials, 7(5):431-456.

Noor, A.K., Burton, S.W., Bert, C.W. (1996). Computational model for sandwich panels and shells. Applied Mechanics Reviews 49(3):155-199.

Rahmani, O., Khalili, S.M.R., Malekzadeh, K. (2010). Free vibration response of composite sandwich cylindrical shell with flexible core, Composite Structures 92:1269-1281.

Rao, M.K., Desai, Y.M. (2004). Analytical solutions for vibrations of laminated and sandwich plates using mixed theory, Composite Structures 63(3-4):361-373.

Reddy, J.N., (2003). Mechanics of laminated composite plates and shells: Theory and analysis. 2nd ed, CRC Press (Boca Raton).

Reddy, J.N., Robbins, D.H. (1994). Theories and computational models for composite laminates, Applied Mechanics Reviews 47:147-165.

Sayyad, A.S., Ghugal, Y.M. (2015). On the free vibration analysis of laminated composite and sandwich plates: A review of recent literature with some numerical results, Composite Structures 129:177-201.

Schwarts-Givli, H., Rabinovitch, O, Frostig, Y. (2007). Free vibrations of delaminated unidirectional sandwich panels with a transversely flexible core-a modified Galerkin approach, Journal of Sound and Vibration 301:253-277.

Vasilive, V.V., Lur'e, S.A. (1992). On refined theories of beams, plates and shells, Journal of Composite Materials 26:422-430. 
Vescovini, R., Dozio, L. (2016). A Variable-Kinematic Model for Variable Stiffness Plates: Vibration and Buckling Analysis, Composite Structures 142:15-26.

Yang, Y., Pagani, A., Carrera, E. (2017). Exact solutions for free vibration analysis of laminated, box and sandwich beams by refined layer-wise theory, Composite Structures 175:28-45.

Zhen W., Wanji, C. (2007). A study of global-local higher-order theories for laminated composite plates, Composite Structures 79:44-54.

Zhen, W., Wanji, C. (2006). Free vibration of laminated composite and sandwich plates using global-local higher-order theory, Journal of Sound and Vibration 298(1-2):333-349. 